$03,09,12,13$

\title{
Спиновая физика экситонов в коллоидных нанокристаллах
}

\author{
(C) А.В. Родина ${ }^{1}$, А.А. Головатенко ${ }^{1}$, Е.В. Шорникова ${ }^{2,3}$, Д.Р. Яковлев ${ }^{2,1}$ \\ ${ }^{1}$ Физико-технический Институт им. А.Ф. Иоффре РАН, \\ Санкт-Петербург, Россия \\ ${ }^{2}$ Experimentelle Physik 2, Technische Universität Dortmund, \\ Dortmund, Germany \\ ${ }^{3}$ Институт фризики полупроводников им. Ржанова, СО РАН, \\ Новосибирск, Россия \\ E-mail: anna.rodina@mail.ioffe.ru
}

\begin{abstract}
Представлен обзор спин-зависимых свойств экситонов в полупроводниковых коллоидных нанокристаллах. Дана сравнительная характеристика фотолюминесценции (ФЛ) нейтральных и заряженых экситонов (трионов). Подробно обсуждаются механизмы и поляризация излучательной рекомбинации „темного“ (запрещенного по спину) экситона, определяющие низкотемпературную ФЛ коллоидных нанокристаллов. Излучательная рекомбинация темного экситона становится возможной в результате одновременного переворота поверхностного спина и спина электрона в темном экситоне, приводящего к подмешиванию состояний светлого экситона. Такой механизм рекомбинации является эффективным в случае неупорядоченного состояния спиновой системы и подавляется при формировании поляронного состояния ферромагнитного характера. Обсуждаются условия и различные механизмы формирования спинового поляронного состояния и возможности его экспериментального детектирования. Представлен обзор экспериментальных и теоретических исследований магнитоиндуцированной циркулярной поляризации ФЛ в ансамблях коллоидных нанокристаллов.
\end{abstract}

Работа выполнена при поддержке Российского Фонда Фундаментальных Исследований (грант № 17-02-01063), Правительства Российской Федерации (постановление № 220, договор № 14.Z50.31.0021, ведущий ученый M.X. Байер) и Deutsche Forschungsgemeinschaft (в рамках проекта ICRC TRR 160).

DOI: $10.21883 /$ FTT.2018.08.46337.07Gr

\section{1. Введение}

Впервые, спектры поглощения и фотолюминесценции (ФЛ) экситонов в полупроводниковых нанокристаллах, распределенных в виде коллоидных частиц в диэлектрической кристаллической среде, исследовались в работе Е.Ф. Гросса и А.А. Каплянского в 1957 году [1]. Систематические исследования эффектов размерного квантования носителей заряда и экситонов и оптических свойств полупроводниковых коллоидных нанокристаллов (nanocrystals (NC)) начались с начала 80x годов прошлого века. В группе А.И. Екимова в ГОИ (Ленинград) исследовались полупроводниковые $\mathrm{NC} \mathrm{CuCl}[2,3]$ и $\mathrm{CdS}$ [4], диспергированные в стеклянной матрице. В Белл лаборатории в США впервые были синтезированы нанокристаллы $\mathrm{CdS}$ в водном растворе [5,6]. Сoвременные технологии позволяют синтезировать полупроводниковые коллоидные нанокристаллы $\mathrm{CdSe}, \mathrm{CdS}$, $\mathrm{ZnSe}, \mathrm{CdTe}, \mathrm{InP}$ и другие, и композитные наноструктуры на их основе различной формы и размера [7], от сферических квантовых точек (quantum dots (QD)) до сверхтонких одномерных стержней (nanorods) и двумерных наноплателетов (nanoplatelets (NPL)) [8] (рис. 1). В настоящее время интерес к исследованиям полупроводниковых коллоидных NC обусловлен как фундаментальным характером описываемых явлений, так и широким спектром и бурным развитием их прак-

тических применений в оптоэлектронике, биологии и медицине [7,9-12].

Особенностью коллоидных $\mathrm{NC}$ является локализация носителей в очень малой области пространства $(1-5 \mathrm{~nm})$, обусловленная размерами структур, а также высокими потенциальными барьерами на их поверхности и разницей диэлектрических констант внутри и снаружи NC. Такая локализация приводит не только к сильной зависимости энергий оптических переходов от

Bare quantum dots

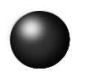

Nanorods

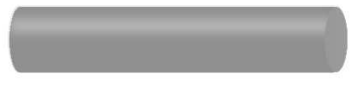

Nanoplatelets

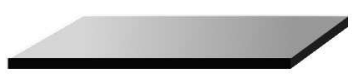

Рис. 1. Многообразие коллоидных нанокристаллов.

Core/shell quantum dots

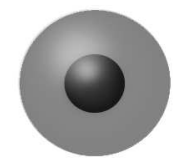

Dot-in-rods

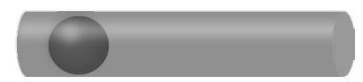

Core/shell nanoplatelets

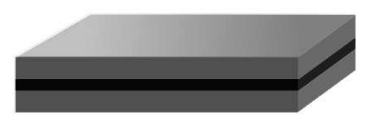

\section{(1)}


размеров, но и к усилению всех взаимодействий между носителями заряда, в том числе спин-зависимых. Наряду с эффектами сильного размерного квантования, оптические и спиновые свойства полупроводниковых NC малого размера определяются взаимодействием носителей с поверхностью, свойства которой зависят от условий их синтеза, стабилизации и пассивации поверхности $[13,14]$. Роль поверхности может проявляться в модификации энергетических спектров носителей, влиянии на излучательную и нерадиационную рекомбинацию экситонов, а также в модификации спиновых и магнитных свойств NC. Для коллоидных NC характерно наличие оборванных связей на поверхности, которые остаются из-за неполной пассивации связей поверхностных ионов лигандами [14] и представляют собой локализованные парамагнитные центры. Намагниченность поверхности QD во внешнем магнитном поле была продемонстритована в SQUID экспериментах $[15,16]$. Эффективным методом характеризации поверхности является метод ядерного магнитного резонанса, усиленный динамической поляризацией ядер на поверхности $[17,18]$.

В работе [19] было показано, что в сферических QD в зависимости от соотношения размеров радиуса нанокристалла $a$ и характерного боровского радиуса экситона в объемном материале $a_{\text {ex }}$ могут реализовываться различные режимы размерного квантования - от предельного случая сильного размерного квантования электронов и дырок $a \ll a_{\mathrm{ex}}$ до предельного случая слабого ограничения $a>a_{\mathrm{ex}}$, при котором экситон квантуется как целое. В режиме сильного размерного квантования край поглощения QD определяется оптическими переходами между уровнями размерного квантования электронов и дырок. При этом экситонные эффекты проявляются только в небольших кулоновских поправках к энергиям перехода [20]. В этом режиме понятие экситона как подвижной квазичастицы, строго говоря, неприменимо, и правильно говорить о возбуждении электрон-дырочной пары [21]. Тем не менее, сильное пространственное ограничение носителей заряда в пределах QD приводит к усилению роли обменных взаимодействий и увеличению расщеплений тонкой структуры экситонов [22,23] по сравнению с объемными материалами и другими низкоразмерными структурами. Поэтому экситонные эффекты, в особенности обменное взаимодействие электрона и дырки, возрастающее как $\propto\left(a_{\text {ex }} / a\right)^{3}$ с уменьшением радиуса QD, становятся критически важными для описания спектров ФЛ. При низких температурах, фотовозбуждение носителей в коллоидных наноструктурах сопровождается их быстрой релаксацией в нижнее энергетическое состояние, которое является оптически пассивным (запрещенным по спину) темным экситоном $|F\rangle$ и сдвинуто по энергии на $\Delta E_{\mathrm{AF}} \approx 1-20 \mathrm{meV}$ от оптически разрешенного светлого экситона $|A\rangle$ [22,24-33]. Однако практически во всех коллоидных структурах, в отличие от большинства эпитаксиальных, излучательная рекомбинация темного экситона оказывается не полностью запрещенной, и, в силу преимущественной заселенности, доминирует в спектрах ФЛ при низких температурах.

Излучательная рекомбинация темного экситона может происходить при наличии вспомогательных процессов, смешивающих темный экситон с одним или несколькими состояниями оптически активного светлого экситона. Смешивание состояний с разной проекцией спина электрона или дырки на ось анизотропии вызывает виртуальный переход темного экситона в состояние светлого экситона, который может излучательно рекомбинировать. Эти процессы медленны по сравнению с прямой оптической рекомбинацией светлых экситонов, и могут быть описаны во втором порядке теории возмущений $[22,28,34,35]$. Свойства низкотемпературной ФЛ зависят от механизма активации излучательной рекомбинации нижнего краевого экситона [35] и определяется спиновой физикой экситонов, в том числе спинзависимым взаимодействием экситонов с поверхностью нанокристаллов.

Мощным инструментом исследования энергетической структуры экситонов и спиновых взаимодействий является поляризационная оптическая спектроскопия [36,37]. Исследования поляризованной ФЛ позволяют получать информацию об анизотропии оптических свойств экситонных состояний, в том числе идентифицировать скрытую анизотропию локализованных экситонов [38]. Поляризационные свойства коллоидных наноструктур определяются несколькими факторами: анизотропией локального электрического поля света, симметрией и поляризацией экситонных состояний и процессами релаксация между ними. Эффект локального поля состоит в перенормировке электрического поля света внутри наноструктуры $\left(\mathbf{E}_{\text {in }}\right)$ относительно электрического поля снаружи $\left(\mathbf{E}_{\text {out }}\right)$, и обусловлен большой разницей (до порядка величины) в диэлектрической проницаемости внутри $\left(\varepsilon^{\text {in }}\right)$ и снаружи $\left(\varepsilon^{\text {out }}\right)$ наноструктуры [39-41]. Поляризационная спектроскопия позволяет идентифицировать поляризацию излучательной рекомбинации темного экситона [27,35,42-44], которая в свою очередь определяется поляризацией светлого экситона, участвующего в ее активации. При анализе оптических, поляризационных и магнитооптических свойств ансамблей коллоидных $\mathrm{NC}$ необходимо учитывать произвольную ориентацию отдельных NC по отношению к направлению распространения света и направлению магнитного поля.

\section{2. Экспериментальные наблюдения излучательной рекомбинации темного экситона}

Одним из проявлений излучательной рекомбинации темного экситона является наличие двух компонент в зависимости интенсивности ФЛ от времени $I(t)$ в экспериментах с временным разрешением в условиях нерезонансного возбуждения при температуре жидкого 
гелия [32,33,45-59]. При этом первая быстрая компонента соответствует релаксации экситона из светлого состояния с энергией $E_{A}$ в темное с энергией $E_{F}=E_{A}-\Delta E_{\mathrm{AF}}$, а вторая медленная компонента соответвует рекомбинации темного экситона со скоростью $\Gamma_{F}$. Именно присутствие медленной компоненты в зависимости $I(t)=\Gamma_{A}^{\mathrm{rad}} N_{A}(t)+\Gamma_{F}^{\mathrm{rad}} N_{F}(t)$ при низких температурах служит доказательством наличия излучательной рекомбинации темного экситона $\Gamma_{F}^{\mathrm{rad}} \neq 0$. Здесь $\Gamma_{A, F}^{\mathrm{rad}}$ и $N_{A, F}$ - скорости излучательной рекомбинации и заселенности светлого и темного экситонов соответственно. Повышение температуры приводит к увеличению заселенности состояний $|A\rangle$ за счет термического заброса из состояний $|F\rangle$, поэтому медленная компонента во временной зависисмости $I(t)$ появляется даже при $\Gamma_{F}^{\mathrm{rad}}=0$. Скорость медленной компоненты $\Gamma_{L}$ возрастает с ростом температуры, а амплитуда быстрой компоненты падает и постепенно исчезает. Анализ температурной зависимости $\Gamma_{L}(T)$ позволяет экспериментально определить энергетическое расщепление $\Delta E_{\mathrm{AF}}[46,47,49,51,58,59]$ из

$$
\begin{aligned}
\Gamma_{L}(T) \approx & \Gamma_{F} \frac{1}{1+\exp \left(-\Delta E_{\mathrm{AF}} / k_{\mathrm{B}} T\right)} \\
& +\Gamma_{A} \frac{\exp \left(-\Delta E_{\mathrm{AF}} / k_{\mathrm{B}} T\right)}{1+\exp \left(-\Delta E_{\mathrm{AF}} / k_{\mathrm{B}} T\right)} .
\end{aligned}
$$

Здесь $\Gamma_{A}-$ скорость рекомбинации светлого экситона, $T$ - температура, $k_{\mathrm{B}}-$ постоянная Больцмана. Выражение (1) предполагает, что на участке времени, когда наблюдается медленный распад ФЛ, установилось термодинамическое равновесие между населенностями $N_{A}$ и $N_{F}$.

Одним из возмущений, смешивающих состояния темного и светлого экситонов, является внешнее магнитное поле, направленное поперечно оси анизотропии $c$ нанокристалла. Уменьшение времени жизни ФЛ темного экситона $\tau \propto 1 / \Gamma_{L}$ во внешнем магнитном поле наблюдались в экспериментах с временным разрешением $[22,56,60]$, и хорошо понято теоретически [22,28,35]. При этом именно возрастание $\Gamma_{L}$ с ростом температуры или внешнего магнитного поля является характерной особенностью ФЛ темных экситонов и позволяет экспериментально отличить ФЛ нейтральных экситонов от ФЛ заряженных экситонов (трионов) в коллоидных NC $[55,59,61,62]$. На рис. 2 показаны типичные экспериментальные зависимости нормализованной интенсивности ФЛ нейтральных экситонов $(a)$ и трионов $(b)$ от времени после импульсного возбуждения из работы [55]. Поскольку все состояния тринов являются оптически активными [63], их смешивание магнитным полем и перераспределение их заселенности с изменением температуры не приводят к ускорению распада ФЛ.

Излучательная рекомбинация темных экситонов непосредственно наблюдалась в спектрах сужения линии люминесценции (fluoresence line narrowing $(\mathrm{FLN})$ ) при резонансном возбуждении ансамбля
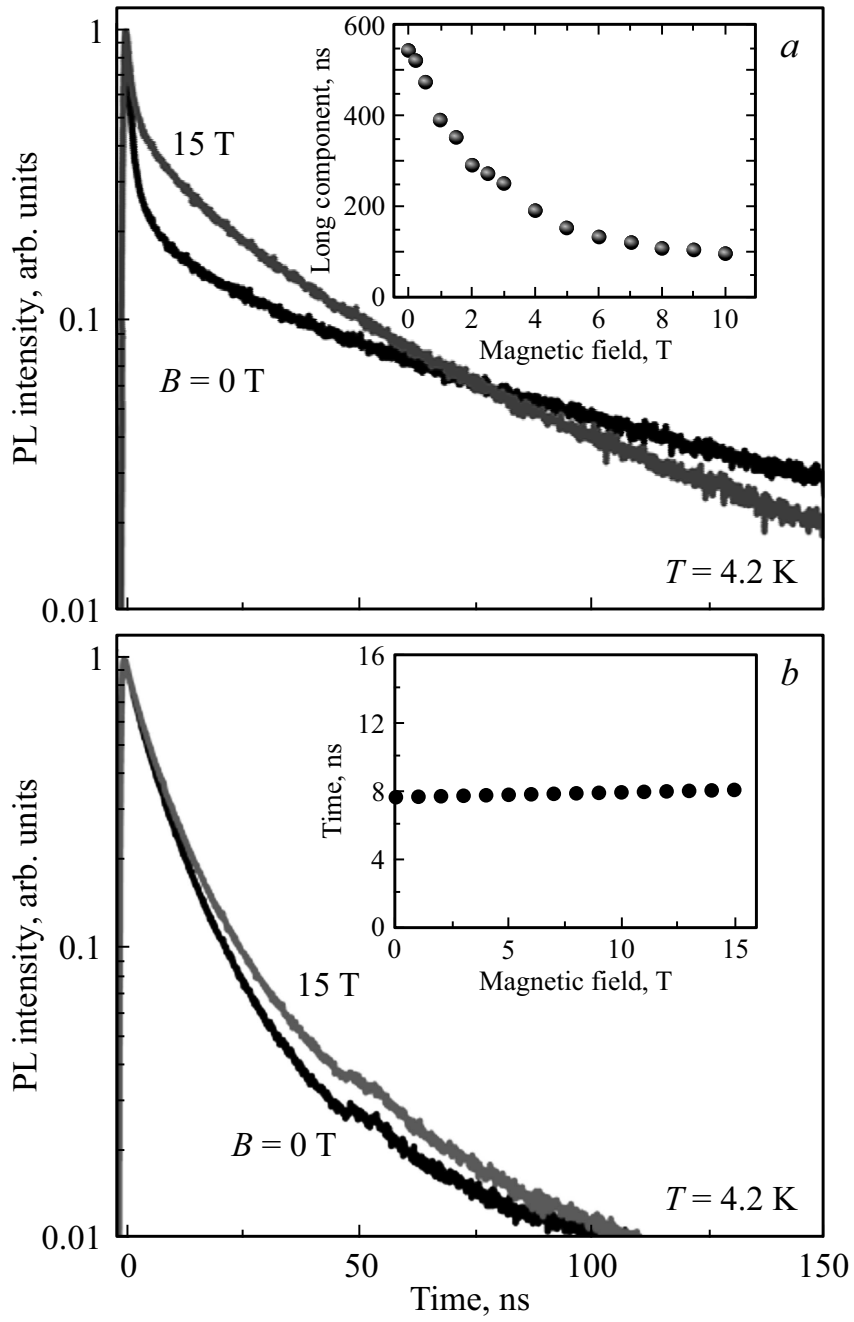

Рис. 2. Экспериментальные зависимости нормализованной интенсивности ФЛ нейтральных экситонов $(a)$ и трионов $(b)$ от времени после импульсного возбуждения в ансамбле сферических нанокристаллов $\mathrm{CdSe} / \mathrm{CdS}$ с радиусом ядра $2.5 \mathrm{~nm}$ и толщиной оболочки $2 \mathrm{~nm}(a)$ и $10 \mathrm{~nm}(b)$ в магнитном поле $B=0$ и 15 Т из работы [55]. На вставках показаны зависимости времени жизни $\tau$ фотолюминесценции (на $(a)$ - долгоживущей компоненты $\propto \Gamma_{L}$ от величины магнитного поля).

NC [24,25,27,42,58,59,64-67], а также в спектрах ФЛ одиночных коллоидных NC [51,52,68-71]. В спектрах FLN ансамбля NC при низких температурах при этом наблюдалась ФЛ темного экситона, состоящая из нескольких линий: бесфононной (zero-phonon line (ZPL)) и сдвинутых на энергию, кратную энергии оптического фонона $E_{\mathrm{LO}}$. Все линии сдвинуты в сторону меньших энергий от энергии возбуждения, причем величина стоксова сдвига ZPL в нанокристаллах малого радиуса нередко оказывается больше, чем величина $\Delta E_{\mathrm{AF}}$, определенная из температурной зависимости $\Gamma_{L}[46,49,50]$. Важно также отметить, что при импульсном возбуждении время жизни ФЛ всех линий в спектре FLN одинаково [58]. Это указывает на то, что наблюдаемые фононные линии 
являются распадом того же самого темного экситонного состояния $|F\rangle$ при помощи оптических фононов, что и линия ZPL. Таким образом, взаимодействие с оптическими фононами является одним из возмущений активирующих излучательную рекомбинацию темного экситона. Взаимодействие с акустическими фононами также может приводить к излучательной рекомбинации темного экситона. Линии, сдвинутые от ZPL на энергию акустических фононов $E_{\mathrm{AC}}$, наблюдались в спектрах одиночных NC, однако не различимы в спектрах FLN.

Вопрос о механизме активации ZPL в нулевом магнитном поле долгое время оставался открытым. Конечное излучательное время жизни состояния темного экситона было получено, например, в расчетах методом псевдопотенциала $[29,72]$ и сильной связи [73]. Было также показано [74], что активация темного экситона может быть обусловлена снижением симметрии коллоидной QD ниже симметрии $C_{6 v}$, при которой она имеет эллипсоидальную форму с осью вращения, направленной вдоль гексагональной оси анизотропии $c$. Дальнейшее понижение симметрии QD приводит к существенной перестройке экситонных состояний [75].

Важный намек на возможный механизм рекомбинации темного экситона был получен группой Бавенди уже в первых работах по резонансному возбуждению ФЛ нанокристаллов $\mathrm{CdSe}[25,26]$. Было обнаружено, что энергия как ZPL, так и первой фононной линии (1PL), сдвигаются на величину примерно $4 \mathrm{meV}$ в сторону энергии возбуждения при повышении температуры от 1.7 до $20 \mathrm{~K}$, и при этом относительная интенсивность ZPL возрастает в 3-4 раза. Заселение состояний светлого экситона при таких температурах в исследовавшихся нанокристаллах радиуса $1.2 \mathrm{~nm} \mathrm{c} \Delta E_{\mathrm{AF}}>14 \mathrm{meV}$ было еще не существенным. Такое же увеличение интенсивности ZPL наблюдалось при приложении внешнего магнитного поля. Однотипное влияние температуры и магнитного поля на рекомбинацию темного экситона позволило предположить, что в основе явления лежит образование и активация экситонного магнитного полярона, аналогичного обнаруженному в полумагнитных полупроводниках [76,77].

До недавнего времени было неясно, какова природа магнитных моментов в коллоидных нанокристаллах $\mathrm{CdSe}$. Спиновое взаимодействие электрона в экситоне с магнитными ионами в легированных Mn квантовых точках исследовалось в работах [78-83]. Магнитные поляроны в нанокристаллах CdSe : Mn наблюдались экспериментально в работах [84-88]. Однако обычные CdSe $\mathrm{NC}$ не содержат магнитных примесей. Взаимодействие с ядерными моментами в CdSe является слабым. Уже в работах $[22,26,45]$ было сделано предположение, что наблюдаемый эффект связан с неспаренными спинами оборванных связей (dangling bond spins (DBS)) на поверхности NC. Теория излучательной рекомбинации рекомбинации темного экситона с одновременным переворотом DBS была развита в работах [34,35]. При этом было предсказано теоретически [34] и обнаружено экспериментально [58] формирование поверхностного магнитного полярона при низких температурах в коллоидных CdSe QD малого размера.

Таким образом, экспериментальные данные указывают на существование нескольких различных механизмов, приводящих к излучательной рекомбинации темного экситона.

\section{3. Энергетическая структура краевого экситона в нанокристаллах и наноплателетах}

В сферических QD прямозонных полупроводников $\mathrm{A}_{\mathrm{II}} \mathrm{B}_{\mathrm{VI}}$ и $\mathrm{A}_{\mathrm{III}} \mathrm{B}_{\mathrm{V}}$ c кубической решеткой основное электронное состояние $1 S_{e}$ дважды вырождено по проекции спина, $s_{z}= \pm 1 / 2$, и описывается волновой функцией $\Psi_{s_{z}}^{e}\left(\mathbf{r}_{e}\right)$. Основное состояние дырки $1 S_{3 / 2}$ четырехкратно вырождено по проекциям $M= \pm 3 / 2, \pm 1 / 2$ полного момента $J=3 / 2$ и описывается волновой функцией $\Psi_{M}^{h}\left(\mathbf{r}_{h}\right)$. В режиме сильного размерного квантования $\left(a \ll a_{\text {ex }}\right)$ и без учета возмущений основное состояние экситона восьмикратно вырожденно и описывается волновой функцией $\Phi$ ехс $\left(\mathbf{r}_{e}, \mathbf{r}_{h}\right)=\Psi^{e}\left(\mathbf{r}_{e}\right) \Psi^{h}\left(\mathbf{r}_{h}\right)$. Вырождение экситонных уровней снимается при учете обменного взаимодействия между электроном и дыркой, а также кристаллического поля и несферичсности формы QD [22].

Электрон-дырочное обменное взаимодействие включает как короткодействующие, так и длиннодействующие вклады $[22,23,28,74,75]$. В QD малого радиуса $a<a_{\mathrm{ex}}$, оба вклада в обменное взаимодействие усиливаются как $\left(a_{\mathrm{ex}} / a\right)^{3}$. Мы рассмотрим далее в деталях вклад короткодействующего обменного взаимодействия, гамильтониан которого имеет вид $[22,89,90]$

$$
\begin{gathered}
H_{\mathrm{eh}}=\left\langle\Phi_{\mathrm{ex}}\left(\mathbf{r}_{e}, \mathbf{r}_{h}\right)\left|\hat{H}_{\mathrm{exch}}\right| \Phi_{\mathrm{ex}}\left(\mathbf{r}_{e}, \mathbf{r}_{h}\right)\right\rangle=-\eta(\boldsymbol{\sigma} \mathbf{J}), \\
\hat{H}_{\mathrm{exch}}=-\frac{2}{3} \varepsilon_{\mathrm{exch}} \nu \delta\left(\mathbf{r}_{e}-\mathbf{r}_{h}\right)(\boldsymbol{\sigma} \mathbf{j}) .
\end{gathered}
$$

Здесь $v$ - объем элементарной ячейки, $\varepsilon_{\text {exch }}-$ константа обменного взаимодействия, $\boldsymbol{\sigma}=\left(\sigma_{x}, \sigma_{y}, \sigma_{z}\right)-$ матрицы Паули, $\mathbf{j}=\left(j_{x}, j_{y}, j_{z}\right)$ и $\mathbf{J}=\left(J_{x}, J_{y}, J_{z}\right)-$ операторы внутреннего момента дырки $j=3 / 2$ и полного момента дырки в экситоне $\mathbf{J}=\mathbf{j}+\mathbf{L}$, где $\mathbf{L}-$ оператор орбитального момента. Усреднение в скобках $\langle>$ ведется только по координатным переменным. Гамильтониан (2) описывает изотропную часть короткодействующего обменного взаимодействия как в объемных полупроводниках, так и в NC произвольной формы. При этом эффективный параметр $\eta$ зависит от вида волновых функций и учитывает перенормировку обменного взаимодействия в NC конкретной формы по сравнению с объемным полупроводником.

В объемном кубическом полупроводнике (например, $\mathrm{CdTe}$ и $c$-CdSe) расщепление между экситонными состояниями с полным моментом $\mathscr{F}=2$ (темное пя- 
тикратновырожденное состояние с энергией, сдвинутой вниз на $-3 \eta / 2)$ и $\mathscr{F}=1$ (светлое трехкратновырожденное состояние с энергией сдвинутой вверх на $+5 \eta / 2)$, равно $\Delta E_{\mathrm{AF}}^{\mathrm{c}}=4 \eta_{\text {bulk }}=8 /(3 \pi) \varepsilon_{\mathrm{exch}}^{c} v_{c} / a_{\mathrm{ex}}^{3}$. В объемном гексагональном полупроводнике со структурой вюртцита (например, $w$-CdSe), кристаллическое поле расщепляет состояния легких и тяжелых дырок по проекции момента $\pm 1 / 2$ и $\pm 3 / 2$ на гексагональную ось $c$ соответвенно. Обменное взаимодействие (2) приводит к расщеплению тяжелого экситона на величину $\Delta E_{\mathrm{AF}}^{w}=3 \eta_{\text {bulk }}=(2 / \pi) \varepsilon_{\mathrm{exch}}^{w} v_{w} / a_{\mathrm{ex}}^{3}$.

$\mathrm{B}$ сферических QD короткодействующее обменное взаимодействие для экситона $1 S_{e} 1 S_{3 / 2}$ может быть найдено как $[22,28]$

$$
\eta=\frac{\Delta_{\mathrm{exch}}}{6 \pi}\left(\frac{a_{0}}{a}\right)^{3} \int_{0}^{\infty} d \tilde{r} \tilde{r}^{2} R_{e}^{2}(\tilde{r})\left[R_{0}^{2}(\tilde{r})+0.2 R_{2}^{2}(\tilde{r})\right],
$$

где $R_{e}$ - радиальная функция основного состояния электрона, $R_{0}$ и $R_{2}$ - радиальные волновые функции, основного состояния дырки, $\tilde{r}=r / a, \Delta_{\text {exch }}=\varepsilon_{\text {exch }} v / a_{0}^{3}-$ перенормированная константа обменного взаимодействия, $a_{0}=1 \mathrm{~nm}$ - единица длины. В случае бесконечного потенциального барьера для носителей на краю нанокристалла безразмерный интеграл, входящий в (3) зависит только от отношения эффективных масс легкой и тяжелой дырок $\beta=m_{l h} / m_{h h}$.

B сферических QD кубических полупроводников расщепление между состояниями экситона с полным моментом $\mathscr{F}=2$ (нижнее) и $\mathscr{F}=1$ равно $4 \eta$, причем $\eta / \eta_{\text {bulk }} \propto\left(a_{\text {ex }} / a\right)^{3}$. Влияние внутреннего кристаллического поля в гексагональных полупроводниках [91] и несферичности формы нанокристалла [92] могут быть учтены в рамках теории возмущений. Совместное действие этих эффектов аналогично действию эффективной деформации $\Delta\left[5 / 4-M^{2}\right] / 2$, расщепляющей основное состояния дырки $1 S_{3 / 2}$ на состояния с проекциями момента $M= \pm 3 / 2$ и $M= \pm 1 / 2$ на ось анизотропии, совпадающей с осью $c$ в гексагональных полупроводниках [22]. Здесь $\Delta=\Delta_{\text {int }}+\Delta_{\text {sh }}, \Delta_{\text {int }}$ описывает эффект кристаллического поля $[91,93]$ и $\Delta_{\mathrm{sh}}-$ эффект несферической формы пространственно-ограничивающего потенциала для дырок. B QD, поверхность которых имеет форму эллипсоида вращения $\left(x^{2}+y^{2}\right) / b^{2}+z^{2} / c^{2}=1$, индуцированное несферичностью расщепление $\Delta_{\text {sh }}$ пропорционально энергии уровня и степени эллиптичности $\mu=c / b-1[92,93]$.

В результате, тонкая структура экситонных уровней в QD описывается гамильтонианом [22]

$$
\hat{H}_{\text {fine }}=-\eta(\boldsymbol{\sigma} \mathbf{J})+\frac{\Delta}{2}\left[5 / 4-M^{2}\right] .
$$

Порядок уровней тонкой структуры экситона зависит от формы QD, как это схематически показано на рис. 3 для CdSe QD. Уровни соответствуют разным проекциям полного углового момента экситона $F$ на ось анизотропии: один уровень с $F= \pm 2$, два уровня с $F= \pm 1$ и два с $F=0$. В случае $\Delta \neq 0$ оба \pm 1 состояния (нижние $1^{L}$ и верхнее $\left.1^{U}\right)$ соответствуют оптически активным светлым экситонам. Для состояний с $F=0$ только верхнее, $0^{U}$, оптически активно, тогда как нижнее, $0^{L}$, отвечает оптически пассивному темному экситону. В сплюснутых QD кубических $(c-\mathrm{CdSe})$ и вюрцитных $(w$-CdSe), а также в сферических $w$-CdSe QD $\Delta>0$ и основное состояние темного экситона соответствует состоянию с $F= \pm 2$ (рис. $3, b)$. В вытянутых $c$-CdSe QD и $w$-CdSe NCs при $\Delta<0$ основное состояние темного экситона соответствует $F=0$ (рис. $3, c$ ).

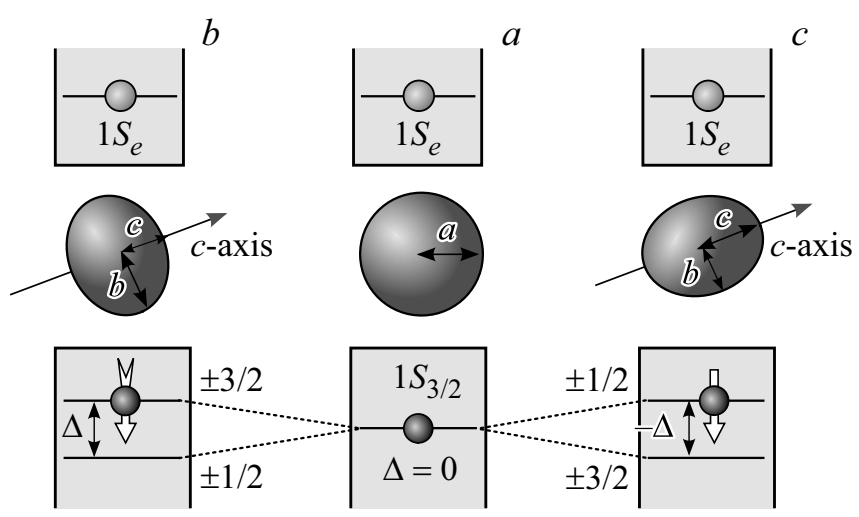

Exciton fine structure

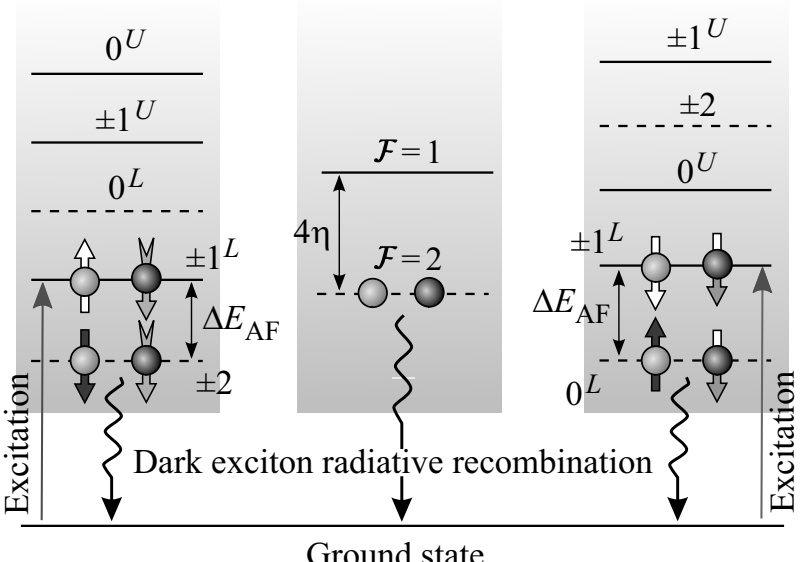

Рис. 3. Нижние уровни размерного квантования электронов и дырок и тонкая структура краевого экситона в нанокристаллах $\mathrm{CdSe}$. (a) Сферический нанокристалл кубического полупроводника радиуса $a$ и квазисферический нанокристалл CdSe co структурой вюрцита. $(b)$ Сплюснутый эллипсоидальный нанокристалл CdSe c „короткой“ полуосью $c<b$. (c) Вытянутые нанокристаллы $\mathrm{CdSe}$ c ,длинной“ полуосью $c>b$. Полная энергия расщепления $\Delta$ определяется кристаллическим полем и анизотропией формы нанокристалла и равна нулю для $(a)$, положительна для $(b)$ и отрицательна для $(c)$. Таким образом, темные экситонные состояния \pm 2 формируются с участием тяжелых дырок $\pm 3 / 2(b)$, в то время как с $0^{L}$ состояние темного экситона формируется с участием легких дырок $\pm 1 / 2$ (c). Состояние с полным моментом $\mathscr{F}=2$ и $\mathscr{F}=1$ в сферических и квазисферических нанокристаллах разделены обменным электрон-дырочным взаимодействием $4 \eta \propto 1 / a^{3}$. Светлые и темные экситонные уровни показаны сплошными и штриховыми линиями соответственно. 

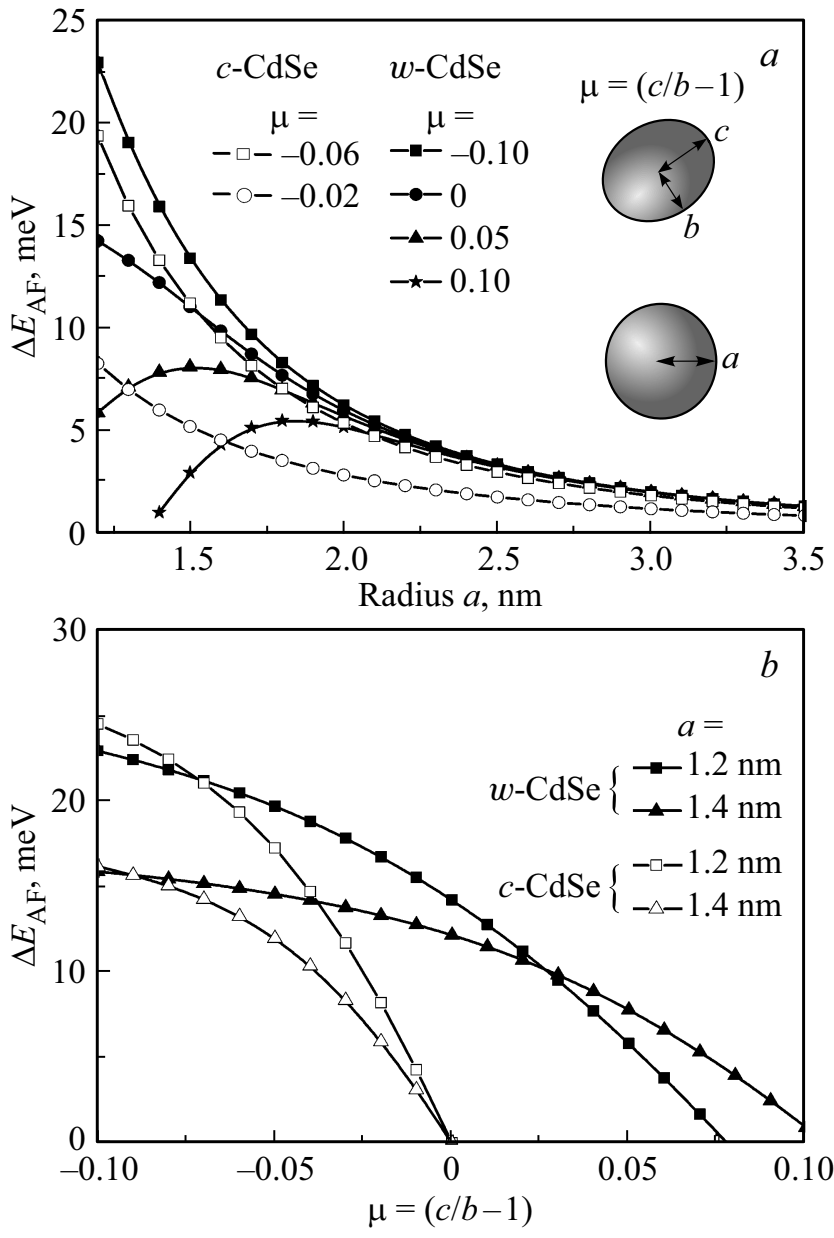

Рис. 4. (a) Зависимость расщепления $\Delta E_{\mathrm{AF}}$ между светлым $\pm 1^{L}$ и темным \pm 2 экситоном от радиуса QD $a$ в $w$-CdSe co структурой вюртцита и кубическом $c$-CdSe при разных значениях параметра несферичности $\mu=(c / b-1)$. (b) Зависимость расщепления $\Delta E_{\mathrm{AF}}$ в QD $w$-CdSe и $c$-CdSe радиуса $a=1.2$ и $1.4 \mathrm{~nm}$ от параметра несферичности $\mu$.

В случае $\Delta>0$, расщепление между уровнями нижнего темного экситона $|F|=2(|F\rangle)$ и нижнего по энергии светлого экситона $1^{L}(|A\rangle)$, равно

$$
\Delta E_{\mathrm{AF}}=2 \eta+\Delta / 2-\sqrt{f^{2}+d},
$$

где $f=(-2 \eta+\Delta) / 2$ и $d=3 \eta^{2}$. Расчеты показывают, что в QD CdSe малого размера энергия расщепления $\Delta E_{\mathrm{AF}}$ составляет 1-20 meV [22]. Вследствие этого только темный экситон вносит вклад в ФЛ при низких температурах. Размерная зависимость величины $\Delta E_{\mathrm{AF}}$ в QD $w$-CdSe со стуктурой вюртцита и кубических $c$-CdSe при разных значениях параметра несферичности $\mu=(c / b-1)$ и зависимость $\Delta E_{\mathrm{AF}}$ в $w$-CdSe и $c$-CdSe QD радиуса $a=1.2$ и $1.4 \mathrm{~nm}$ от $\mu$ показаны на рис. 4. Для расчетов использовались значения константы обменного взаимодействия $\varepsilon_{\text {exch }}^{w}=320 \mathrm{meV}$ соответсвующее экспериментальному значению $\Delta E_{\mathrm{AF}}^{w}=0.13 \mathrm{meV}$ из работы [94] и $\varepsilon_{\text {exch }}^{c}=160 \mathrm{meV}$, соответвующее предположению $\Delta_{\text {exch }}^{\mathrm{c}}=\Delta_{\text {exch }}^{w}[59]$. В случае $\eta \gg \Delta$ расщепление определяется анизотропным расщеплением дырок как $\Delta E_{\mathrm{AF}}=3 \Delta / 4$. В противоположном случае $\eta \ll \Delta$ расщепление определяется обменным взаимодействием как $\Delta E_{\mathrm{AF}}=3 \eta$.

Именно этот последний случай реализуется в двумерных коллоидных наноплателетах [59]. При этом $\Delta E_{\mathrm{AF}}=3 \eta_{2 \mathrm{D}}$ может быть найдено в результате усреднения гамильтониана (2) с волновой функцией экситона

$$
\Phi_{\mathrm{ex}}\left(\mathbf{r}_{e}, \mathbf{r}_{h}\right)=\Psi_{\mathrm{CM}}(\rho) \Psi\left(\rho_{e}-\rho_{h}\right) \psi_{e}\left(z_{e}\right) \psi_{h}\left(z_{h}\right),
$$

где одномерные функции $\psi_{e}$ и $\psi_{h}$ описывают размерное квантования электрона и дырки вдоль анизотропной оси $z \| c$ NPL, и двумерные волновые функции $\Psi_{\mathrm{CM}}(\rho)$ и $\Psi\left(\rho_{e}-\rho_{h}\right)$ описывают движение центра масс экситона и относительное движение электрона и дырки в экситоне в плоскости NPL соответственно. Для бесконечно больших потенциальных барьеров на поверхности плателета

$$
\Delta E_{\mathrm{AF}}=3 \eta_{2 \mathrm{D}}=\Delta_{\mathrm{exch}}|\tilde{\Psi}(0)|^{2} / \tilde{L}
$$

Здесь $\tilde{L}=L / a_{0}, L-$ толщина NPL, $\tilde{\Psi}(0)=\Psi(0) a_{0}$. Расчет размерной зависмости $\Delta E_{\mathrm{AF}}$ с $\varepsilon_{\text {exch }}^{c}=160 \mathrm{meV}$ и учетом разницы диэлектрических констант $\varepsilon_{\text {in }}=6$ и $\varepsilon_{\text {out }}=2$ дает хорошее согласие с экспериментальными данными для $c$-CdSe [59].

\section{4. Излучательная рекомбинация краевого экситона}

4.1. Правила отбора для оптических переходов в дипольном приближении. В дипольном приближении взаимодействие экситона со светом описывается возмущением $e /\left(m_{0} \omega\right) \mathbf{E} \hat{\mathbf{p}}$, где $e-$ заряд и $m_{0}-$ масса свободного электрона, $\mathbf{E}=E\left[\exp (-i \omega t) \mathbf{e}+\exp (i \omega t) \mathbf{e}^{*}\right]-$ электрическое поле световой волны, $E$ - амплитуда электрического поля, $\omega$ и е - частота и поляризация света $[95,96]$. Матричные элементы оператора импульса $\mathbf{d}=\langle G|\hat{\mathbf{p}}| X\rangle$ между основным состоянием $|G\rangle \mathrm{NC}$ и краевым экситонным состоянием $|X\rangle$ будем называть далее дипольными элементами перехода. Они связаны с матричными элементами $\tilde{\mathbf{d}}=\langle G|e \hat{\mathbf{r}}| X\rangle$ дипольного оператора $e \hat{\mathbf{r}}[96]$ как $\mathbf{d}=i m_{0}\left(E_{\mathrm{g}} / e \hbar\right) \tilde{\mathbf{d}}$, где $E_{\mathrm{g}}$ - ширина запрещенной зоны полупроводника. Ненулевыми дипольными элементами обладают только остояния светлого экситона $|X\rangle= \pm 1^{L, U}, 0^{U}$ :

$$
d_{0}=\left\langle G\left|\hat{p}_{z}\right| 0^{U}\right\rangle=\frac{P K}{\sqrt{3}},
$$

$$
\begin{aligned}
& d_{ \pm 1}^{U}=\frac{1}{2}\left\langle G\left|\hat{p}_{x} \pm i p_{y}\right| \pm 1^{U}\right\rangle=\mp \frac{d_{0}}{2 \sqrt{2}}\left(C^{+}+\sqrt{3} C^{-}\right) \\
& d_{ \pm 1}^{L}=\frac{1}{2}\left\langle G\left|\hat{p}_{x} \pm i p_{y}\right| \pm 1^{L}\right\rangle=\frac{d_{0}}{2 \sqrt{2}}\left(\sqrt{3} C^{+}-C^{-}\right),
\end{aligned}
$$


где $K=\int d^{3} \mathbf{r} \Phi_{\mathrm{exc}}(\mathbf{r}, \mathbf{r}), P$ - матричный элемент оператора $\hat{\mathbf{p}}$ между блоховскими функциями зоны проводимости и валентной зоны, и

$$
C^{ \pm}=\sqrt{\frac{\sqrt{f^{2}+d} \pm f}{2 \sqrt{f^{2}+d}}} .
$$

В случае $\Delta=0$, коэффициенты $C^{+}=1 / 2$ и $C^{-}=\sqrt{3} / 2$, так что экситоны $\pm 1^{L}$ являются темными.

Состояние светлого экситона $0^{U}$ поляризовано вдоль оси $c$ NC. Линейно поляризованный диполь часто называют невырожденным одномерным диполем [97]. Состояния светлого экситона $\pm 1^{U, L}$ поляризованы перпендикулярно к оси $c$ и известны как вырожденные двумерные диполи [97-99]. Скорость спонтанной излучательной рекомбинации экситонных состояний $|X\rangle= \pm 1^{L, U}, 0^{U}$ может быть найдена как [35]

$$
\begin{aligned}
& \frac{1}{\tau_{0^{U}}}=\frac{D_{\|}}{\tau_{0}}, \quad \frac{1}{\tau_{1}, L}=\frac{D_{\perp}}{\tau_{0}} \frac{\left|d_{1^{U, L}}\right|^{2}}{\left|d_{0}\right|^{2}}, \\
& \frac{1}{\tau_{0}}=\frac{4 e^{2} \omega n}{3 \hbar m_{0}^{2} c^{3}}\left|d_{0}\right|^{2}=\frac{16 e^{2} \omega n}{9 \hbar m_{0}^{2} c^{3}} P^{2} K^{2} .
\end{aligned}
$$

Здесь $n$ - коэффициент преломления света в среде, окружающей $\mathrm{NC}, c-$ скорость света, $D_{\perp}=\left|E_{x, y}^{\text {in }}\right|^{2} /\left|E_{x, y}^{\text {out }}\right|^{2} \quad$ и $\quad D_{\|}=\left|E_{z}^{\text {in }}\right|^{2} /\left|E_{z}^{\text {out }}\right|^{2}-$ факторы экранирования локального поля, зависящие от направления поля относительно оси анизотропии [39-41,44,100]. Для сферических QD $D_{\perp} \approx D_{\|}=D$. При этом для одномерных структур, нанородов и структур ядро-нанород $D_{\perp} \ll D_{\|}$, что приводит к усилению оптических переходов с участием $0^{U}$ экситона $[31,43,44]$. Для двумерных NPL, $D_{\perp} \gg D_{\|}$.

4.2. Механизмы излучательной рекомбинации темного экситона. Механизмы рекомбинации темных экситонов для всех возможных структур уровней, показанных на рис. 3, рассмотрены в деталях в работе [35]. Эти механизмы должны обеспечить подмешивание состояний светлого экситона $\pm 1^{L, U}, 0^{U}$ к состояниям темного экситрона $\pm 2,0^{L}$ либо через переворот спина электрона, вызванный какимлибо возмущением $\hat{V}_{e}$, либо через подмешивание дырочных состояний, вызванное возмущением $\hat{V}_{h}$. За подмешивание состояний отвечают четыре матричных элемента: $V_{e}=\left\langle\Psi_{+1 / 2}^{e}\left|\hat{V}_{e}\right| \Psi_{-1 / 2}^{e}\right\rangle$ описывает переворот спина электрона, а элементы $V_{l h}=\left\langle\Psi_{+1 / 2}^{h}\left|\hat{V}_{h}\right| \Psi_{-1 / 2}^{h}\right\rangle$, $V_{h, 1}=\left\langle\Psi_{+3 / 2}^{h}\left|\hat{V}_{h}\right| \Psi_{+1 / 2}^{h}\right\rangle$ и $V_{h, 2}=\left\langle\Psi_{+3 / 2}^{h}\left|\hat{V}_{h}\right| \Psi_{-1 / 2}^{h}\right\rangle-$ смешивание волновых функций основного состояния дырки, $\Psi_{M}^{h}$, с начальным и конечным значением проекции $M$, отличающимися на \pm 1 или \pm 2 . Ненулевые матричные элементы $V_{e}, V_{l h}$ и $V_{h, 1}$ могут быть обеспечены внешним магнитным полем, нормальным к оси $c$ [22,28], или обменным взаимодействием со спинами оборванных связей на поверхности нанокристалла [34,35]. Взаимодействие с оптическими и акустическими фононами с угловым моментом 2 обеспечивают смешивание дырочных состояний, описываемое операторами

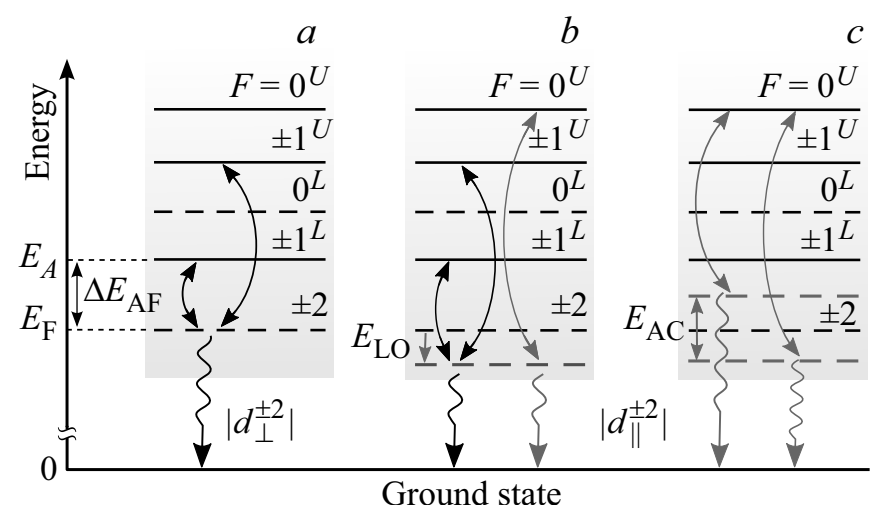

Рис. 5. Схема тонкой структуры краевого экситона и излучательного распада темного экситона \pm 2 , активированного внешним магнитным полем или спинами оборванных связей на поверхности нанокристалла $(a)$, или взаимодействием с оптическими $(b)$ и акустическими $(c)$ фононами. Уровни светлых (оптически разрешенных) и темных (запрещенных) экситонов показаны сплошными и штриховыми линиями соответственно. Возможное подмешивание уровней темного и светлых экситонов показаны круглыми стрелками. Поляризация излучательной рекомбинации темного экситона зависит от типа диполя оптически активных уровней, подмешанных к темному экситону. Подмешивание состояний $\pm 1^{L, U}\left(0^{U}\right)$ приводит к появлению ненулевых дипольных элементов $\left|d_{\perp}^{ \pm 2}\right|\left(\left|d_{\|}^{ \pm 2}\right|\right)$.

$V_{h, 1}$ and $V_{h, 2}$ [91,101,102]. Кроме того, диагональные матричные элементы $V_{\uparrow(\downarrow), M}=\left\langle\Psi_{\uparrow(\downarrow), M}\left|\hat{V}_{e}+\hat{V}_{h}\right| \Psi_{\uparrow(\downarrow), M}\right\rangle$ возмущений $\hat{V}_{e}$ и $\hat{V}_{h}$, например, вызванных внешним магнитным полем, кристаллическим полем или механическим напряжением вдоль оси $c$, могут приводить к активации $\pm 1^{L}$ и $0^{L}$ экситонов путем подмешивания состояний $\pm 1^{U}$ и $0^{U}$ экситонов без переворота спина электрона или дырки. Смешивание экситонов $\pm 1^{U}$ и $\pm 1^{L}$ при $\Delta \neq 0$ представляет собой известный механизм такой активации.

Вероятность излучательной рекомбинации темного экситона может быть найдена в рамках теории возмущений второго порядка [22,28,34,35], которая описывает виртуальные переходы темного экситона в состояние светлого экситона $\pm 1^{L, U}, 0^{U}$, за которыми следует их излучательный распад. Для состояний $|X\rangle= \pm 2$ и $0^{L}$ темного экситона, дипольные элементы перехода в первом порядке теории возмущения равны нулю. Однако подмешивание светлого $\pm 1^{L, U}\left(0^{U}\right)$ экситона может приводить к ненулевым дипольным элементам $\left|d_{\perp}^{ \pm 2}\right|$ и $\left|d_{\perp}^{0 L}\right|\left(\left|d_{\|}^{ \pm 2}\right|\right.$ и $\left.\left|d_{\|}^{0 L}\right|\right)$ соотвественно во втором порядке теории возмущений. Поляризация излучения темного экситона определяется поляризационными свойствами соответствующего светлого экситона.

Актуальные механизмы подмешивания и поляризация излучения для темного экситона $F= \pm 2$ показаны на рис. 5. ФЛ темного экситона, активированная с помощью оптических фононов, может быть поляризована как вдоль так и поперечно к оси $c$ и сдвинута от ZPL на 
энергию $E_{\mathrm{LO}}$. При низких температурах может наблюдаться только стоксова компонента LO-индуцированной ФЛ (рис. 5,b). ФЛ темного экситона, активированная с помощью акустических фононов, в сферических нанокристаллах поляризована преимущественно вдоль оси $c$ и сдвинута от ZPL на энергию $E_{\mathrm{AC}}$, причем могут наблюдаться обе, стоксова и антистоксова, компоненты (рис. 5,c). Активация ФЛ вследствии анизотропии в плоскости, поперечной оси $c$, рассмотренная в [74], имеет такие же поляризационные характеристики, как и ФЛ с помощью акустических фононов. Далее мы остановимся подробнее только на механизмах излучения темного экситона $F= \pm 2$ через подмешивание светлых экситонов $\pm 1^{L, U}$, дающего вклад в ZPL (рис. 5, $a$ ). Такое подмешивание может происходить во внешнем магнитном поле, имеющем ненулевую компоненту перпендикулярно оси $c$, или за счет одновременного переворота спина электрона и спина оборванной связи на поверхности (DBS).

4.3. Излучательная рекомбинация темного экситона $F= \pm 2$ во внешнем магнитном поле. При наличии внешнего магнитного поля $\mathbf{B}=\left(B_{x}, B_{y}, B_{z}\right)$, возмушение описывается членами $\hat{V}_{e}=\frac{1}{2} g_{e} \mu_{\mathrm{B}}\left(\boldsymbol{\sigma}^{e} \mathbf{B}\right)$ и $\hat{V}_{h}=-g_{h} \mu_{\mathrm{B}}(\mathbf{J B}) \quad[22]$, где $g_{e, h}-$ эффективные $g$-факторы основного состояния электрона и дырки соответственно и $\mu_{\mathrm{B}}-$ магнетон Бора. Диагональные матричные элементы $V_{\uparrow, M}=\left(g_{e} / 2-M g_{h}\right) \mu_{\mathrm{B}} B \cos \theta$ и $V_{\downarrow, M}=-\left(g_{e} / 2+M g_{h}\right) \mu_{\mathrm{B}} B \cos \theta$, где $\theta-$ угол между направлением магнитного поля с осью $c$, приводят к зеемановскому расщеплению экситонных состояний $[22,28]$. В случае, когда зеемановские энергии $g_{e} \mu_{\mathrm{B}} B$ и $g_{h} \mu_{\mathrm{B}} B$ намного меньше $\Delta E_{\mathrm{AF}}$, только матричный элемент $V_{e}=g_{e} \mu_{\mathrm{B}}\left(B_{x}-i B_{y}\right) / 2$, отвечающий за переворот спина электрона, приводит к появлению ненулевого $\left|d_{\perp}^{ \pm 2}\right|[35]$. Скорость излучательной рекомбинации, рассчитанная во втором порядке теории возмущений, имеет вид [35]

$$
\Gamma_{F}^{B}=\frac{\left|V_{e}\right|^{2}}{12 \eta^{2}} \frac{D_{\perp}}{\tau_{0}}=\frac{\left(g_{e} \mu_{\mathrm{B}} B \sin \theta\right)^{2}}{48 \eta^{2}} \frac{D_{\perp}}{\tau_{0}}
$$

Следует отметить, что выражение для индуцированной магнитным полем скорости излучательной рекомбинации темного экситона в QD малого радиуса, полученное впервые в рамках теории возмущений второго порядка в работе [22] (см. также выражение (3.46) в работе [28]) содержит ошибку. Оно предполагает возможность излучения темного экситона за счет матричного элемента оператора $V_{h}$, отвечающего за смешивание легких и тяжелых дырок. Однако, как показано в работе [35], такое смешивание приводит к излучательной рекомбинации со скоростью $\propto(B \sin \theta)^{4}$ только в случае, когда зеемановские энергии сравнимы с $\Delta E_{\mathrm{AF}}$. Из выражения (13) видно, что в пределе $\mu_{\mathrm{B}} B \ll \Delta E_{\mathrm{AF}}$ излучательное время темного экситона не зависит от величины расщепления $\Delta$ между состояниями $\pm 1 / 2$ и $\pm 3 / 2$ легких и тяжелых дырок. При этом, величина $\Delta E_{\mathrm{AF}}$ зависит от обоих парамеров тонкой структуры $\eta$ и $\Delta$.
В двумерных NPL обменное взаимодействие между электроном и дыркой намного меньше расщепления легких и тяжелых дырок $\Delta$, и $\Delta E_{\mathrm{AF}}=3 \eta_{2 D}$. В результате, индуцированная магнитным полем скорость излучательной рекомбинации имеет вид:

$$
\Gamma_{F}^{B}=\frac{\left(g_{e} \mu_{\mathrm{B}} B \sin \theta\right)^{2}}{4 \Delta E_{\mathrm{AF}}} \Gamma_{A} .
$$

В случае, когда зеемановская энергия сопоставима с $\Delta_{\mathrm{AF}}$, рассмотрение смешанного состояния темного и нижнего светлого экситонов в NPL приводит к более общему выражению

$$
\Gamma_{F}^{B}=\frac{\sqrt{1+\zeta^{2}+2 \xi \cos \theta}-1-\zeta \cos \theta}{\sqrt{1+\zeta^{2}+2 \xi \cos \theta}} \frac{\Gamma_{A}}{2},
$$

где $\xi=\mu_{\mathrm{B}} g_{e} B /\left(\Delta E_{\mathrm{AF}}\right)$. Это выражение совпадает с выражением для скорости радиационной рекомбинации темного экситона в QD большого размера при $\mu_{\mathrm{B}} B \approx 3 \eta \ll \Delta$, полученным в работе [22] (см. также выражение (3.51) в работе [28]). В этом приближении $\Delta E_{\mathrm{AF}}=3 \eta$ и $\Gamma_{A}=3 / 4 \tau_{0}$. Легко показать, что выражение (15) переходит в (14) при $\xi^{2} \sin ^{2} \theta \ll 1$.

4.4. Излучательная рекомбинация темного экситона $F= \pm 2$ : взаимодействие с оборванными связями на поверхности. Рассмотрим взаимодействие экситонов с DBS на поверхности нанокристалла, приводящее к активации темного экситона $F= \pm 2$. В сферических QD, можно рассматривать только взаимодействие электрона с поверхностью, так как дырка дополнительно локализована в центре QD благодаря кулоновскому взаимодействию с электроном. Для электрона, гамильтониан обменного взаимодействия может быть записан как $H_{s s}=\sum_{j} H_{s s}^{j}$, где суммирование ведется по всем оборванным связям и

$$
\begin{gathered}
H_{s s}^{j}=\left\langle\Phi_{\mathrm{ex}}\left(\mathbf{r}_{e}, \mathbf{r}_{h}\right)\left|\hat{H}_{e}^{j}\right| \Phi_{\mathrm{ex}}\left(\mathbf{r}_{e}, \mathbf{r}_{h}\right)\right\rangle=-\alpha_{j}\left(\boldsymbol{\sigma} \boldsymbol{\sigma}^{j}\right), \\
\hat{H}_{e}^{j}=-v \tilde{\alpha} \delta\left(\mathbf{r}_{e}-\mathbf{R}_{j}\right)\left(\boldsymbol{\sigma} \boldsymbol{\sigma}^{j}\right) .
\end{gathered}
$$

Здесь $\boldsymbol{\sigma}^{j}$ и $\mathbf{R}_{j}-$ вектора матриц Паули и координаты $j$-той оборванной связи и $\tilde{\alpha}-$ константа обменного взаимодейтсвия в элементарной ячейке, $\alpha_{j}=\tilde{\alpha} \nu \int\left|\Phi_{\mathrm{ex}}\left(\mathbf{r}_{e}, \mathbf{r}_{h}\right)\right|^{2} \delta\left(\mathbf{r}_{e}-\mathbf{R}_{j}\right) d \mathbf{r}_{e} d \mathbf{r}_{h}$, и усреднение в скобках \langle\rangle ведется только по координатным переменным. В сферических QD обменное взаимодействие электрона в основном $1 S_{e}$ состоянии со всеми оборванными связями одинаково: $\alpha_{j}=\alpha=\tilde{\alpha} \nu\left|R_{e}(a)\right|^{2}$, где $\left|R_{e}(a)\right|^{2}$ - квадрат волновой функции электрона на поверхности NC. Для двумерных NPL предполагалось [103], что оборванные связи находятся на плоских поверхностях, и что эффективный боровский радиус двумерного экситона намного меньше длины локализации экситона в плоскости. В этом случае эффективная константа взаимодействия $\alpha_{j}=v \tilde{\alpha}\left|\psi_{e}( \pm L / 2)\right|^{2}\left|\Psi_{C M}\left(\rho_{j}\right)\right|^{2}$ $=\alpha_{2 D}\left|\Psi_{C M}\left(\rho_{j}\right)\right|^{2}$, где $\left|\Psi_{C M}\left(\rho_{j}\right)\right|^{2}-$ вероятность нахождения экситона в точке локализации $\rho_{j}$ оборванной связи. Константа $\alpha_{2 D}$ имеет размерность $\mathrm{meV} \cdot \mathrm{nm}^{2}$ и одинакова для всех DBS. 

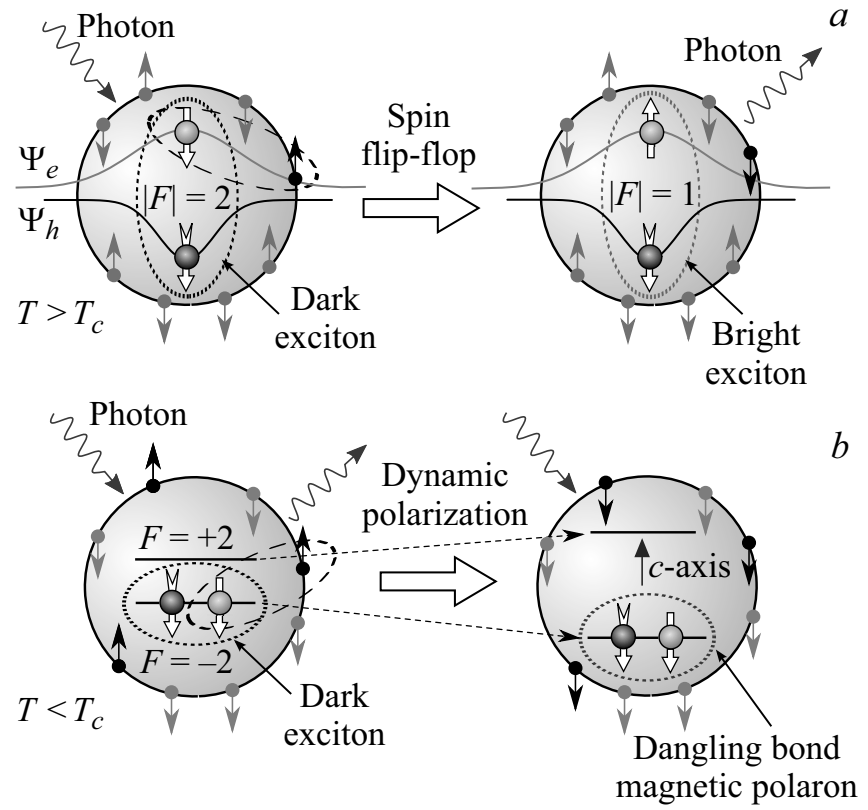

Рис. 6. (a) Схематическое изображение процесса излучательной рекомбинации темного экситона в результате переворота спина оборванной связи на поверхности QD. $(b)$ Процесс динамической поляризации спинов и формирования магнитного полярона при температурах ниже критической $T_{c}$.

Обменное поле DBS действует на электрон в экситоне и может приводить к энергетическому расщеплению экситонного состояния $\Delta E_{\mathrm{ex}}=2 E_{p} \rho_{\mathrm{db}}$ и его поляризации $\rho_{\text {ex }}$ даже в нулевом магнитном поле $[34,103]$. Здесь $E_{p}=\alpha N_{\mathrm{db}}$ для $\mathrm{QD}$, где $N_{\mathrm{db}}-$ полное число $\mathrm{DBS}$ на поверхности, и $E_{p}=\alpha N_{\mathrm{db}}=2 \alpha_{2 D} n_{\mathrm{db}}$ для NPL, где $N_{\mathrm{db}}=2 n_{\mathrm{db}} S_{\mathrm{loc}}$ - число DBS, с которыми взаимодейтсвует экситон в двумерном NPL, $n_{\mathrm{db}}$ - плотность DBS на поверхности, $S_{\mathrm{loc}}=1 / \int\left|\Psi_{C M}(\rho)\right|^{4} d^{2} \rho-$ эффективная площадь локализации экситона, и $\alpha=\alpha_{2 \mathrm{D}} / S_{\mathrm{loc}}$. Поляризация DBS определена как разность между числом спинов $N_{\mathrm{db}}^{+}$, ориентированных параллельно, и числом спинов $N_{\mathrm{db}}^{-}$, ориентированных антипараллельно оси $z \| c$ :

$$
\rho_{\mathrm{db}}=-\frac{\sum_{j}\left\langle\hat{\sigma}_{z}^{j}\right\rangle}{N_{\mathrm{db}}}=\frac{N_{\mathrm{db}}^{-}-N_{\mathrm{db}}^{+}}{N_{\mathrm{db}}} .
$$

При произвольной ориентации DBS $\rho_{\mathrm{db}}=0$. Однако изза стохастических флуктуаций, среднее значение квадрата спина оборванных связей $\sum_{j} s^{j}\left(s^{j}+1\right)=3 N_{\mathrm{db}} / 4$. Флуктуационное обменное поле DBS всегда имеет ненулевую компоненту, поперечную оси $c$, и, как и внешнее магнитное поле, активирует излучательную рекомбинацию темного экситона. Возмущение $\hat{V}_{e}=\hat{V}_{e}^{+}+\hat{V}_{e}^{-}$, где $\hat{V}_{e}^{ \pm}=\hat{A}^{\mp} \sigma_{ \pm}=-(1 / 2) \sum_{j} \alpha_{j} \sigma_{\mp}^{j} \sigma_{ \pm}$, где $\sigma_{ \pm}=\sigma_{x} \pm \sigma_{y}$, может вызвать спонтанный переворот проекции спина электрона и проекции спина $j$-ой оборванной связи на поверхности, сопровождающийся виртуальным переходом темного экситона в светлое состояние с его последующей излучательной рекомбинацией. Такой процесс схематически показан на рис. 6, (a). Суммирование по всем DBS позволяет получить скорость рекомбинации $\Gamma_{F, \pm 2}^{\mathrm{db}}$ для уровней \pm 2 :

$$
\begin{gathered}
\text { QD : } \quad \Gamma_{F, \pm 2}^{\mathrm{db}}=2 \gamma_{\mathrm{ex}} N_{\mathrm{db}}^{\mp}, \quad \gamma_{\mathrm{ex}}=\left(\frac{\alpha}{\eta}\right)^{2} \frac{D_{\perp}}{6 \tau_{0}}, \\
\mathrm{NPL}: \quad \Gamma_{F, \pm 2}^{\mathrm{db}}=2 \gamma_{\mathrm{ex}} N_{\mathrm{db}}^{\mp}=4 \gamma_{\mathrm{ex}}^{2 D} n_{\mathrm{db}}^{\mp}, \\
\gamma_{\mathrm{ex}}^{2 D}=\left(\frac{\alpha_{2 D}}{\eta}\right)^{2} \frac{2 \Gamma_{A}}{9 S_{\mathrm{loc}}}=\frac{\gamma_{\mathrm{ex}}}{S_{\mathrm{loc}}}
\end{gathered}
$$

Рекомбинация наиболее эффективна и имеет максимальную скорость $\Gamma_{F, \pm 2}^{\mathrm{db}}=\Gamma_{\mathrm{db}}^{c}=\gamma_{\mathrm{ex}} N_{\mathrm{db}}$ при произвольной ориентации DBS. Рекомбинация становится спинзависимой в случае возникновения ненулевой поляризации $\rho_{\mathrm{db}} \neq 0$. При наличии в то же время поляризации темного экситона $\rho_{\mathrm{ex}}=\left(N_{\mathrm{ex},-2}-N_{\mathrm{ex},+2}\right) / N_{\mathrm{ex}}$, где $N_{\mathrm{ex}}=\left(N_{\mathrm{ex},-2}+N_{\mathrm{ex},+2}\right)-$ средняя заселенность экситонных состояний в $\mathrm{NC}$, скорость излучательной рекомбинации $\Gamma_{F}^{\mathrm{db}}=\left(\Gamma_{F,+2}^{\mathrm{db}} N_{\mathrm{ex},+2}+\Gamma_{F,-2}^{\mathrm{db}} N_{\mathrm{ex},-2}\right) / N_{\mathrm{ex}}$ имеет вид

$$
\Gamma_{F}^{\mathrm{db}}=\gamma_{\mathrm{ex}} N_{\mathrm{db}}\left[1-\rho_{\mathrm{db}} \rho_{\mathrm{ex}}\right]=\Gamma_{\mathrm{db}}^{c}\left[1-\rho_{\mathrm{db}} \rho_{\mathrm{ex}}\right] .
$$

Таким образом, излучательная рекомбинация подавляется при формировании поляронного состояния с $\rho_{\mathrm{db}} \rho_{\mathrm{ex}}>0$.

\section{5. Динамическая поляризация поверхностных спинов и магнитный полярон}

В работе [34] была предложена теоретическая модель формирования экситонного магнитного полярона на спинах оборванных связей (dangling bond magnetic polaron $(\mathrm{DBMP}))$ в результате динамической поляризации DBS в процессе оптического возбуждения и рекомбинации темного экситона, схематически показанной на рис. $6, b$. Согласно этой модели, возникающая в результате флуктуации $\rho_{\mathrm{db}} \neq 0$ приводит к $\rho_{\mathrm{ex}} \neq 0$. При условии $\alpha>0$ рекомбинация поляризованного экситона через переворот DBS увеличивает $\rho_{\mathrm{db}}$. Существует критическая температура $T_{c}$, ниже которой описанная положительная обратная связь приводит к формированию комбинированной магнитной поляризации нанокристалла с $\rho_{\mathrm{db}} \rho_{\mathrm{ex}} \neq 0$.

Теоретическая модель магнитного полярона - области с ориентированными в одном направлении спинами носителей и локализованных магнитных моментов - была предложена более полувека назад в работаx $[104,105]$. Флуктуационные и коллективные магнитные поляроны, формирующиеся и поддерживающиеся за счет энергии обменного взаимодействия, широко исследовались в полумагнитных полупроводниках и низкоразмерных гетероструктурах на их основе $[77,83,106]$ и наблюдались экспериментально в коллоидных нанокристаллах $\mathrm{CdSe}: \mathrm{Mn}$ [84-88]. Возможность динамической 
самополяризации ядерных спинов в полупроводниках с помощью линейно поляризованной оптической накачки была предложена в работе М.И. Дьяконова и В.И. Переля [107]. При этом ядерная намагниченность возникает только при температурах ниже $1 \mathrm{~K}$, а электронная спиновая система остается неполяризованной. В работе [108] теоретически предложен механизм динамической самополяризации ядер в квантовых точках, основанный на рекомбинации темных экситонов, сопровождающейся переворотом спина ядра. Этот механизм не требует низкой температуры решетки, однако предполагает равную заселенность спиновых подуровней экситонов и не приводит к формированию экситонного магнитного полярона. Предложенный в работе [34] механизм динамической поляризации DBS имеет общие черты с обеими моделями ядерной динамической самополяризации $[107,108]$. Существенные отличия предложенной в [34] модели состоят в том, что (i) поляризация DBS возникает именно из-за разности заселенностей спиновых подуровней темного экситона $F= \pm 2$ и (ii) приводит к формированию магнитного полярона. Критическая температура формирования полярона $T_{c}$ прямо пропорциональна энергии полярона $E_{p}=\alpha N_{\mathrm{db}}$ (равной полной энергии обменного взаимодействия при полной поляризации DBS $\left.\rho_{\mathrm{db}}=1\right)$ и достигает $T_{c} \approx 20-40 \mathrm{~K}$ в QD малого размера. В отличие от коллективного обменного магнитного полярона в полумагнитных полупроводниках, в том числе в легированных Mn QD [87], формирование DBMP происходит в процессе оптического возбуждения и рекомбинации темного экситона, поэтому его можно назвать динамическим магнитным поляроном.

Связь между поляризацией $\mathrm{DBS} \rho_{\mathrm{db}}$ и поляризацией экситона $\rho_{\mathrm{ex}}$ дается в общем случае двумя условиями $[34,58]$

$$
\rho_{\mathrm{ex}}=\tanh \frac{\Delta E_{\mathrm{ex}}}{2 k_{\mathrm{B}} T}=\tanh \frac{\alpha N_{\mathrm{db}} \rho_{\mathrm{db}}}{k_{\mathrm{B}} T},
$$

и

$$
\rho_{\mathrm{db}}=\frac{\rho_{\mathrm{ex}}+\chi \tanh \left(\alpha \rho_{\mathrm{ex}} / k_{\mathrm{B}} T\right)}{1+\chi_{\mathrm{eff}}} .
$$

Выражение (22) описывает термодинамически равновесную поляризацию экситона в эффективном обменном поле, созданном поляризованными DBS. Такая поляризация возникает за время спиновой релаксации $\tau_{s}$ между расщепленными подуровнями, которое намного меньше времени жизни темного экситона $\tau$. Выражение (23) описывает два вклада поляризации экситона в поляризацию DBS: динамический и термодинамический. Динамический вклад связан с переворотом одного DBS со скоростью $2 \gamma_{\text {ex }}$ в процессе рекомбинации экситона. Термодинамический вклад связан с релаксацией DBS со скоростью $\gamma_{\mathrm{db}}$ к термодинамически равновесному положению в эффективном обменном поле экситона. Параметры $\chi=\gamma_{\mathrm{db}} / 2 \gamma_{\mathrm{ex}}$ и $\chi_{\text {eff }}$ описывают отношение этих скоростей в фотовозбужденных $\mathrm{NC}$ в присутсвии экситона и усредненное по всему ансамблю, соотвественно. При этом были рассмотрены две предельные ситуации: $\chi_{\mathrm{eff}}=\chi / N_{\mathrm{ex}}$ (когда релаксация DBS происходит во всех NC с одиноковой скоростью) и $\chi_{\text {eff }}=\chi$ (когда релаксация поляризации DBS к нулю в невозбужденных NC происходит намного медленнее, чем к равновесной поляризации в присутствии экситона). Эти два предельных режима релаксации DBS предсказывают существенно различную зависимость максимально достижимой поляризации $\rho_{\mathrm{db}}$ и критической температуры $T_{c}$ от средней населенности экситонов в NC и, следовательно, от мощности возбуждения [34,58]. Критическая температура $T_{c}$ динамического формирования DBMP

$$
T_{c} \approx \frac{\alpha N_{\mathrm{db}}}{k_{\mathrm{B}}\left(1+\chi_{\mathrm{eff}}\right)}=\frac{E_{p}}{k_{\mathrm{B}}\left(1+\chi_{\mathrm{eff}}\right)}
$$

соотвествует условию существования нетривиального совместного решения уравнений (22) и (23) при $\alpha>0$. При этом в предельном случае $\chi \gg 1$ динамическим вкладом можно пренебречь и рассмотреть формирование обменного термодинамического полярона. Оно возможно при любом знаке $\alpha$ при температурах ниже

$$
T_{c}^{\text {th }} \approx \frac{|\alpha| \sqrt{N_{\mathrm{db}}}}{k_{\mathrm{B}}}=\frac{\left|E_{p}\right|}{k_{\mathrm{B}} \sqrt{N_{\mathrm{db}}}} .
$$

Важно, что формирование поляронного состояния при понижении температуры ниже критической подавляет процесс излучательной рекомбинации темного экситона \pm 2 и приводит к понижению энергии оптического перехода на энергию полярона $E_{p} \rho_{\mathrm{db}}$. Это делает возможным оптическое детектирование данного эффекта в низкотемпературных спектрах спектрах FLN при резонансном возбуждении. В работе [34] было показано, что модель DBMP позволяет объяснить наблюдавшуюся ранее в работах [25,109] температурную зависимость стоксова сдвига ZPL в QD малого радиуса и ее отсутсвие в QD большого радиуса, так как $T_{c} \propto E_{p}$ убывает с радиусом QD.

Детальные исследования зависимостей спектров FLN нанокристаллов CdSe радиусом $1.4 \mathrm{~nm}$, синтезированных двумя различными методами с различной пассивацией поверхности, от температуры, мощности и длительности возбуждения позволили однозначно экспериментально доказать формирование DBMP и определить его параметры [58]. При этом была обнаружена медленная динамика как формирования окончательной поляризации DBS в ансамбле QD, так и их медленная релаксация к неупорядоченному состоянию после выключении возбуждения. Анализ спектров, полученных при длительном (более двух часов) непрерывном возбуждении позволил показать, что понижение температуры приводит к увеличению стоксовых сдвигов ФЛ бесфононной $S_{\mathrm{ZPL}}=E_{A}-E_{\mathrm{ZPL}}$ и первой фононной линий $S_{1 \mathrm{PL}}=E_{A}-E_{1 \mathrm{PL}}$ прямо пропорционально уменьшению отношения их интенсивностей $I_{\mathrm{ZPL}} / I_{1 \mathrm{PL}}$. В случае формирования полярона эти зависимости контроллируются 


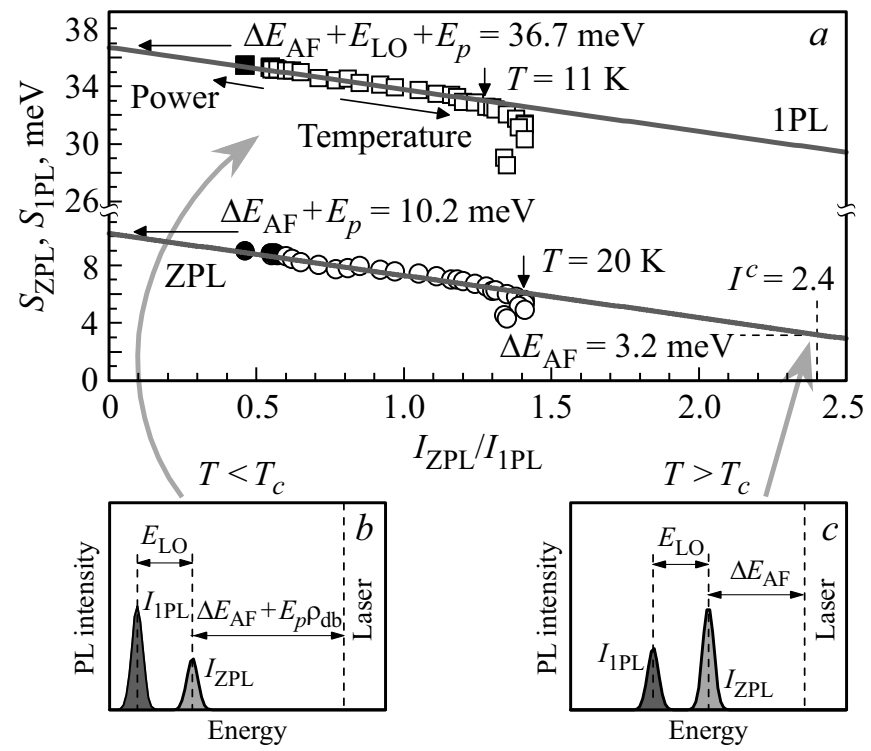

Рис. 7. Теоретическое моделирование и определение параметров полярона в нанокристаллах $\mathrm{CdSe}$ радиусом $1.4 \mathrm{~nm}$ из работы [58]. (a) Параметрические зависимости стоксова сдвига бесфононной линии $E_{\mathrm{ZPL}}$ и первой фононной линии $E_{1 \mathrm{PL}}$ от отношения их интенсивностей $I_{\mathrm{ZPL}} / I_{1 \mathrm{PL}}$. Символы отвечают экспериментальным данным из температурной зависимости спектров FLN, измеренным после двух часов освещения. Сплошные прямые - теоретический расчет с помощью уравнений $(26) .(b)$ и $(c)$ : Схематическое положение и относительные интенсивности линий ZPL и 1PL при температурах ниже и выше критической температуры $T_{c}$ соотвественно.

величиной поляризации спинов поверхностных связей $\rho_{\mathrm{db}}$ и параметрически связаны между собой как

$$
S_{1 \mathrm{PL}}=E_{1}-E^{c} \frac{I_{\mathrm{ZPL}}}{I_{1 \mathrm{PL}}}, \quad S_{\mathrm{ZPL}}=E_{\mathrm{sat}}-E^{c} \frac{I_{\mathrm{ZPL}}}{I_{1 \mathrm{PL}}} .
$$

Здесь $E_{1}=E_{p}+E_{\mathrm{AF}}+E_{\mathrm{LO}}=E_{\mathrm{sat}}+E_{\mathrm{LO}} \quad$ и $\quad E^{c}=E_{p} I^{c}$, $I^{c}=I_{1 \mathrm{PL}} / I_{\mathrm{ZPL}}$ при $T=T_{c}$. На рис. 7 показаны параметрические зависимости сдвига бесфононной и первой фононной линии как функции отношения интенсивностей из работы [58]. Линейная аппроксимация экспериментальных данных дает значения $E_{\mathrm{sat}}=E_{p}+$ $+\Delta E_{\mathrm{AF}} \approx 10.2 \mathrm{meV}, E_{\mathrm{LO}}=E_{1}-E_{\mathrm{sat}}=26.5 \pm 0.5 \mathrm{meV}$ и $E^{c} \approx 2.9 \mathrm{meV}$. Таким образом, важные соотношения между параметрами полярона могут быть получены непосредственно из параметрических зависимостей, показанных на рис. 7, без детального восстановления температурной зависимости $\rho_{\mathrm{db}}$. Более того, экспериментальные зависимости на рис. 7 параметризованы изменением поляризации $\rho_{\mathrm{db}}$ не только с температурой, но и с увеличением мощности возбуждения. Увеличение скорости генерации экситонов приводит к увеличению стоксова сдвига и уменьшению относительной интенсивности ZPL, однако наблюдаемый эффект значительно меньше, чем это предсказывает приближение $\chi_{\mathrm{eff}}=\chi / N_{\mathrm{ex}}$. Кроме того, дополнительные исследования уменьшения стоксова сдвига после выключении источника возбуждения свидетельствуют об очень медленной (порядка 4 часов) релаксации DBS в невозбужденных нанокристаллах [58]. Поэтому, для детального анализа температурных зависимостей $\rho_{\mathrm{db}}(T)$ и $\rho_{\mathrm{ex}}(T)$ в работе [58] использовалось приближение $\chi_{\mathrm{eff}}=\chi$. Была определена энергия полярона $E_{p}=7 \mathrm{meV}$ при $\alpha=0.12 \mathrm{meV}, N_{\mathrm{db}}=60$. Одновременно, с тем же набором параметров удалось описать измеренную зависимость медленной компоненты распада ФЛ $\Gamma_{L}$ (рис. 8) с помощью уравнения (1), в котором была учтена зависимость скорости рекомбинации темного эксито-

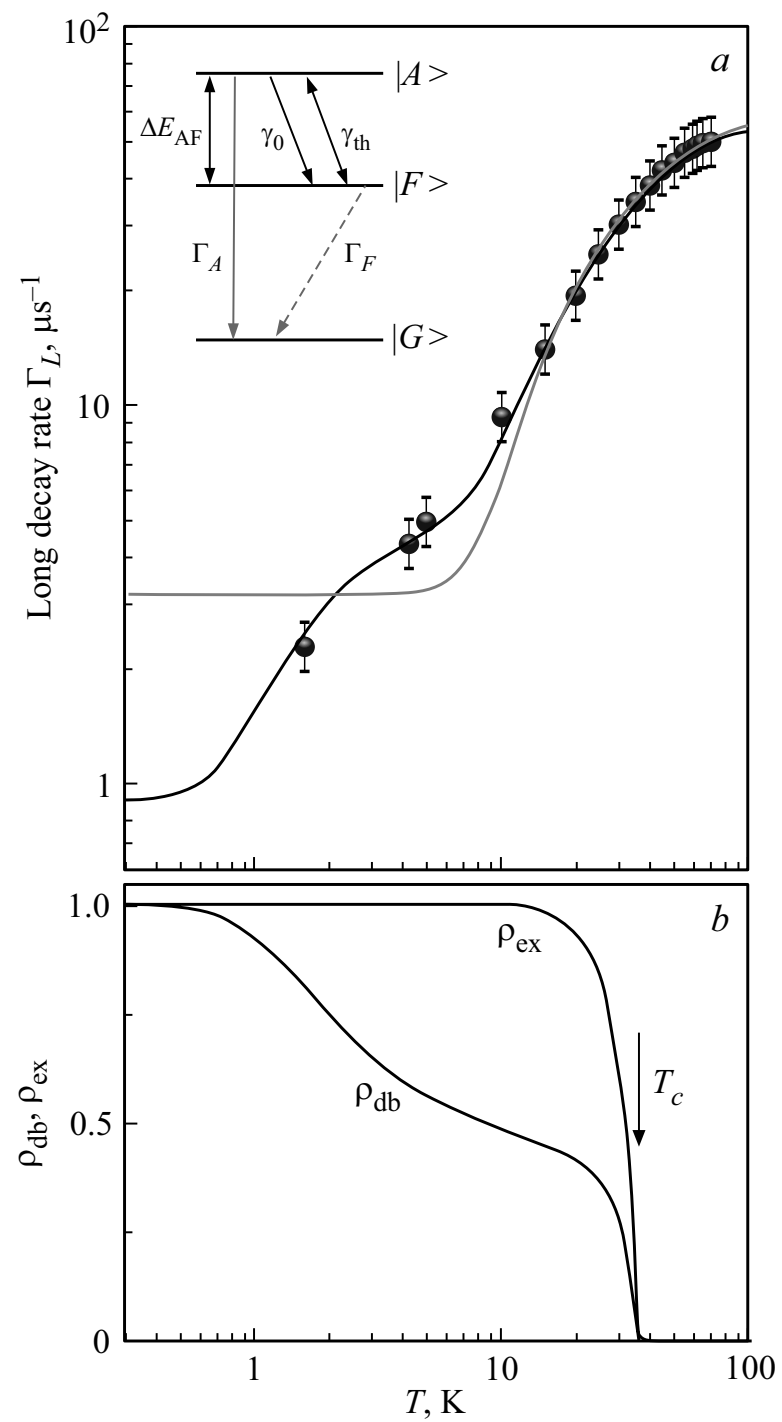

Рис. 8. (a) Изменение $\Gamma_{L}$ в нанокристаллах $\mathrm{CdSe}$ радиуса $1.4 \mathrm{~nm} \mathrm{c}$ температурой. Символами показаны экспериментальные данные из работы [58], сплошными кривыми - результаты теоретического расчета по формуле (1) с $\Delta E_{\mathrm{AF}}=3.2 \mathrm{meV}$ в предположении $\Gamma_{F}=$ const без учета (серая кривая) и $\Gamma_{F}(T)=\Gamma_{\mathrm{LO}}+\Gamma_{\mathrm{db}}^{c}\left[1-\rho_{\mathrm{db}}(T) \rho_{\mathrm{ex}}(T)\right]$ с учетом (черная кривая) активации излучательной рекомбинации темного экситона при подавлении поляронного состояния. На вставке показана схема уровней и оптических переходов светлого и темного экситонов. $(b)$ Изменение поляризаций $\rho_{\mathrm{ex}}$ и $\rho_{\mathrm{db}}$ с температурой. Критическая температура формирования полярона составляет $T_{c} \approx 35 \mathrm{~K}$. 
на от температуры $\Gamma_{F}(T)=\Gamma_{\mathrm{LO}}+\Gamma_{\mathrm{db}}^{c}\left[1-\rho_{\mathrm{db}}(T) \rho_{\mathrm{ex}}(T)\right]$. Таким образом, удалось согласованно определить величину расщепления между темным и светлым экситоном $\Delta E_{\mathrm{AF}}=3.2 \mathrm{meV}$ из спектров сужения ФЛ и экспериментов с временным разрешением. Для найденных параметров критическая температура термодинамического формирования обменного полярона $T_{c}^{\text {th }} \approx 11 \mathrm{~K}$ в три раза меньше чем критическая температура динамического формирования $T_{c} \approx 35 \mathrm{~K}$.

Формирование DBMP при низких температурах и его подавление с ростом температуры объясняет температурные зависимости спектров резонансного возбуждения FLN как проявление магнитных свойств немагнитных CdSe QD малого размера. Важно отметить, что метод резонансного возбуждения позволил также детектировать формирование экситонного магнитного полярона в CdSe : Mn QD [87]. Усиление обменного взаимодействия в коллоидных $\mathrm{NC}$ создает благоприятные условия для спонтанного намагничивания спинов $\mathrm{Mn}$ и формирования коллективного экситонного магнитного полярона в нулевом магнитном поле. При этом, в отличие от ранее наблюдавшихся поляронов в полумагнитных полупроводниках, обнаруженный полярон в нулевом магнитном поле характеризуется почти полной поляризацией магнитных моментов $\mathrm{Mn}$. В отличие от DBMP, магнитный полярон в $\mathrm{CdSe}: \mathrm{Mn}$ формируется благодаря обменному взаимодействию за время меньшее времени жизни экситона и полностью разрушается в отсутсвие экситона. Поэтому включение внешнего магнитного поля, которое выстраивает спины $\mathrm{Mn}$ до возбуждения экситона, драматически влияет на спектры FLN. Температурная зависимость спектров FLN нанокристаллов CdSe : Mn также меняется при приложении внешнего магнитного поля [87].

Подчеркнем, что в немагнитных $\mathrm{CdSe}$ QD магнитный полярон создается при возбуждении неполяризованным светом. В каждой из QD магнитный момент ориентирован вдоль оси $c$ данной QD. Эти магнитные моменты, однако, не приводят к возникновению макроскопического магнитного момента ансамбля случайно ориентированных QD и появлению циркулярной поляризации ФЛ. Однако присутствие DBS на поверхности, как и присутствие ионов Mn в полумагнитных структурах, может влиять и на степень циркулярной поляризации ФЛ в магнитном поле. Изменение знака циркулярной поляризации в легированных $\mathrm{Mn}$ наноплателетах $\mathrm{CdSe} / \mathrm{CdMnS} / \mathrm{CdS}$ наблюдалось в работе [110]. Влияние обменного поля DBS, поляризованных во внешнем магнитном поле, на степень циркулярной поляризации ФЛ в немагнитных CdSe NPL обсуждается теоретически в работе [103] и в настоящее время исследуется нами экспериментально. Обменное поле DBS может быть также ответсвенно за наблюдавшееся экспериментально противоречие между знаком циркулярной поляризации ФЛ и зависящим от поля энергетическим сдвигом поляризованных компонент в измерениях на ансамблях QD $[48,56,66]$. Обменное поле DBS может играть роль внутреннего магнитного поля, ответственного за увеличение времени жизни темного экситона при приложении малых магнитных полей, наблюдавшееся в исследованиях одиночных QD в работе [52].

\section{6. Циркулярная поляризация фотолюминесценции нейтральных и заряженных экситонов}

Изучение степени циркулярной поляризации ФЛ является эффективным методом исследования спиновых подуровней экситонов и экситонных комплексов, их заселенности и спиновой динамики (энергетической релаксации между подуровнями). Циркулярная поляризация ФЛ может возникнуть в результате оптической накачки - возбуждения циркулярно поляризованным светом - или при приложении внешнего магнитного поля. В первом случае разность заселенностей спиновых подуровней создается оптической накачкой, однако наблюдение циркулярной поляризации ФЛ возможно только при условии, что время спиновой релаксации $\tau_{s}$ между подуровнями больше, чем время жизни $\tau$. Это условие очень сложно выполнить для долгоживущих темных экситонов в коллоидных NC. Во втором случае разность заселенностей зеемановских подуровней создается в результате энергетического расщепления между ними в магнитном поле. Теория формирования циркулярной поляризации экситонной ФЛ в магнитном поле и обзор ее экспериментальных исследований в различных полупроводниковых гетероструктурах представлен в работе [37]. Здесь мы остановимся подробнее только на особенностях, характерных для коллоидных NC.

6.1. Циркулярная поляризация ФЛ темного экситона Как уже обсуждалось выше, низкотемпературная ФЛ нейтральных коллоидных $\mathrm{NC}$ определяется излучательной рекомбинацией темного экситона. Энергия зеемановского расщепления экситонных уровней в магнитном поле обычно намного меньше чем $\Delta E_{\mathrm{AF}}$. Поэтому степень циркулярной поляризации ФЛ зависит в первую очередь от расщепления $\Delta E_{\text {ex }}$ и поляризации $\rho_{\text {ex }}$ темного экситона, а также механизма его излучательной рекомбинации. Расщепление $\Delta E_{\mathrm{ex}}$, как и расщепление уровней тяжелой дырки $\Delta E_{h h}$, определяющее циркулярную поляризацию отрицательно заряженного триона, зависят от угла $\theta$ между направлением оси анизотропии $c$ структуры и направлением приложенного магнитного поля $\mathbf{B}$.

Теория циркулярной поляризации ФЛ от ансамбля произвольно ориентированных QD была впервые развита в работе [45]. При этом предполагалось, что рекомбинация темного экситона \pm 2 полностью наследует поляризационные свойства светлого экситона \pm 1 . В этом случае измеряемую в геометрии Фарадея степень циркулярной поляризации ФЛ $P_{c}(B)$ можно найти в 
результате интегрирования по всем возможным ориентациям как [45]

$$
\begin{aligned}
P_{c}(B) & =\frac{I^{+}-I^{-}}{I^{+}+I^{-}}=\frac{\int_{0}^{1}\left[I^{+}(x)-I^{-}(x)\right] d x}{\int_{0}^{1}\left[I^{+}(x)+I^{-}(x)\right] d x} \\
& =-\frac{\int_{0}^{1} \rho_{\mathrm{ex}}(B, x) 2 x \rho d x}{\int_{0}^{1}\left(1+x^{2}\right) d x} .
\end{aligned}
$$

Здесь $\rho_{\mathrm{ex}}(B, x)$ - поляризация экситона в магнитном поле, зависящая от величины и направления магнитного поля, $x=\cos \theta, I^{ \pm}-$интенсивности света, измеряемые в $\sigma^{ \pm}$поляризации при постоянном или импульсном возбуждении. При этом равновесная степень циркулярной поляризации ФЛ $P_{c}^{\mathrm{eq}}$, измеряемая на временах $t \gg \tau_{s}$ после импульсного возбуждения, определяется термодинамически равновесной заселенностью спиновых подуровней и $\rho_{\mathrm{ex}}(B, x)=\rho_{\mathrm{ex}}^{\mathrm{eq}}=\tanh \Delta E_{\mathrm{ex}}(B, x) / 2 k_{\mathrm{B}} T$. Напротив, интегральная по времени поляризация $P_{c}^{\text {int }}$ (совпадающая с $P_{c}^{c w}$, измеряемой при постоянном возбуждении), зависит дополнительно от величины динамического фактора $d=\tau /\left(\tau+\tau_{s}\right): \rho_{\mathrm{ex}}^{\mathrm{int}}=d \rho_{\mathrm{ex}}^{\mathrm{eq}}, P_{c}^{\mathrm{int}}(B)=d P_{c}^{\mathrm{eq}}(B)$. В экспериментальных исследованиях магнитоциркулярной поляризации нейтральных экситонов наблюдалось быстрое нарастание $P_{c}(t)$ со временем спиновой релаксации темного экситона $\tau_{s} \ll \tau[43,45,48,55,56]$, и значения $P_{c}^{c w}(B)=P_{c}^{\text {int }}(B) \approx P_{c}^{\mathrm{eq}}(B)$.

Выражение (27) предсказывает максимальное значение циркулярной поляризации $\left|P_{c}^{\mathrm{sat}}\right|=\left|P_{c}^{\mathrm{eq}}(B \rightarrow \infty)\right|=$ $=0.75$. В случае низкого квантового выхода ФЛ, зависимость скорости излучательной рекомбинации от $x$ приводит к $\left|P_{c}^{\text {sat }}\right|=0.66$ [45]. Такое же (вплоть до $\left.\left|P_{c}^{\text {sat }}\right|=0.66\right)$ понижение можно ожидать в случае селективного возбуждения нанокристаллов с гексагональной осью с, ориентированной перпендикулярно внешнему магнитному полю, например при резонансном возбуждении экситонов $0^{U}$ [43]. Эффект селективного возбуждения в геометрии Фарадея описывается фактором $P_{\mathbf{k}}(x)=0.5\left(D_{\|}+D_{\perp}\right)\left[1-\sigma x^{2}\right]$ под интегральными выражениями в числителе и знаменателе (27), где $\sigma=\left(1-D_{\perp}\right) /\left(1+D_{\perp}\right)[43,44]$. В случае резонансного возбуждения экситонов \pm 1 , селективное возбуждение QD с гексагональной осью $\mathbf{c} \| \mathbf{B}$, увеличит максимальное значение до $\left|P_{c}^{\text {sat }}\right|=0.8$. В ряде экспериментальных исследований, однако, наблюдались значения $\left|P_{c}^{\mathrm{sat}}\right| \ll 0.75[43,48,56]$. Для объяснения наблюдавшихся значений $\left|P_{c}^{\text {sat }}\right|=0.35$ в ансамбле QD CdTe [56] было сделано предположение о сосуществовании вытянутых и сплюснутых QD в ансамбле. При этом в вытянутых QD $\mathrm{CdTe}$ основным состоянием экситона является темный экситон $F= \pm 2$, а в сплюснутых - темный экситон $F=0^{L}[22]$. Поскольку состояние $F=0^{L}$ не вырождено по спину, ФЛ из сплюснутых CdTe QD неполяризована и ее вклад уменьшает величину $\left|P_{c}^{\text {sat }}\right|$.

Модель циркулярной поляризации темного экситона $F= \pm 2$ может быть обобщена с учетом различных механизмов активации его излучательной рекомбинации. Поляризационные свойства темного экситона $F= \pm 2$ могут определяться не только свойствами светлого экситона $F= \pm 1$, но и приобретать свойства светлого экситона $0^{U}$ (рис. 5). С учетом дипольных элементов $\left|d_{\perp}^{ \pm 2}\right|$ и $\left|d_{\|}^{ \pm 2}\right|$ и анизотропии факторов экранирования локального поля $D_{\perp}$ и $D_{\|}$, выражения для вероятностей $W^{ \pm 2}\left(\sigma^{ \pm}\right)$, описывающих вклад излучательной рекомбинации подуровней $F= \pm 2$ в интенсивности $I^{ \pm}$, имеют вид

$$
\begin{aligned}
& W^{ \pm 2}\left(\sigma^{+}\right)=D_{\perp} \frac{\left|d_{\perp}^{ \pm 2}\right|^{2}}{2}(1+\cos \theta)^{2}+D_{\|} \frac{\left|d_{\|}^{ \pm 2}\right|^{2}}{2} \sin ^{2} \theta, \\
& W^{ \pm 2}\left(\sigma^{-}\right)=D_{\perp} \frac{\left|d_{\perp}^{ \pm 2}\right|^{2}}{2}(1-\cos \theta)^{2}+D_{\|} \frac{\left|d_{\|}^{ \pm 2}\right|^{2}}{2} \sin ^{2} \theta .
\end{aligned}
$$

В работе [43] анализировалось выражение для равновесной циркулярной поляризации, полученное в предположении $\left|d_{\perp, \|}^{+2}\right|=\left|d_{\perp, \|}^{-2}\right|$ :

$$
P_{c}^{\mathrm{eq}}(B)=-\frac{\int_{0}^{1}\left|d_{\perp}^{ \pm 2}\right|^{2} 2 x \tanh \left(\Delta E_{\mathrm{ex}} / 2 k_{\mathrm{B}} T\right) P_{\mathbf{k}}(x) d x}{\int_{0}^{1}\left[\left|d_{\perp}^{ \pm 2}\right|^{2}\left(1+x^{2}\right)+R\left|d_{\|}^{ \pm 2}\right|^{2}\left(1-x^{2}\right)\right] P_{\mathbf{k}}(x) d x},
$$

где $R=D_{\|} / D_{\perp}$. Выражение (29) позволило объяснить уменьшение максимального значения $\left|P_{c}^{\text {sat }}\right|$ от ансамбля композитных $\mathrm{CdSe} / \mathrm{CdS} \mathrm{NC}$ ядро-нанород (dot-in-rod), состоящих из сферического ядра $\mathrm{CdSe}$, введенного в стержни $\mathrm{CdS}$, с ростом отношения длины стрежня $l$ к его ширине $d$. В таких структурах дырки локализованы в сферическом ядре $\mathrm{CdSe}$, форма и размер которого определяет тонкую структуру экситона. В то же время, анизотропия локального электрического поля определяется геометрией нанорода $\mathrm{CdS}$, вытянутого вдоль гексагональной оси $c$. С ростом отношения $d / l$ возрастает фактор $R$ и роль оптических переходов, поляризованных вдоль оси $c$.

Как обсуждалось в разделе 4.4, скорость рекомбинации с экситонных подуровней $F=-2$ и $F=2$ становится различной в случае возникновения поляризации DBS $\rho_{\mathrm{db}} \neq 0:\left|d_{\perp, \|}^{+2}\right| \neq\left|d_{\perp, \|}^{-2}\right|$. При этом обменное поле поляризованных DBS также будет влиять на расщепление спиновых подуровней $F=-2$ и $F=2$ в магнитном поле и на их заселенности. Эти два эффекта вместе могут приводить к нелинейной зависимости степени циркулярной поляризации от магнитного поля в ансамбле коллоидных NC [103]. В настоящее время нами ведутся теоретические и экспериментальные исследования влияния спинов поверхностных связей на степень циркулярной поляризации экситонной ФЛ в коллоидных наноструктурах. 
6.2. Спиновая динамика отрицательно заряженных трионов. Как уже обсуждалось в разделе 2, исследование рекомбинациной динамики ФЛ позволяет различить рекомбинацию нейтральных и заряженных экситонов (трионов). Независимость времени жизни ФЛ $\tau$ от магнитного поля (типичный пример показан на рис. $2, b$ ) позволила сделать выводы о трионной природе ФЛ в сферических QD [55] и кубических $\mathrm{CdSe} / \mathrm{CdS}$ NPL [62] с большой толщиной оболочки. В обоих случаях, отрицательный знак циркулярной поляризации в магнитном поле соответсвует рекомбинации отрицательно заряженного триона. Соответсвующая схема уровней и оптических переходов показана на вставке на рис. 9, $a$. Основное состояние отрицательного триона состоит из двух электронов в синглетном состоянии и дырки с проекцией момента $M= \pm 3 / 2$ на ось квантования. Экспериментальная зависимость равновесной степени циркулярной поляризации $P_{c}^{\mathrm{eq}}(B)$ хорошо описывается выражением (27), в котором равновесная поляризации $\rho_{\mathrm{ex}}=\rho_{h h}^{\mathrm{eq}}(B, x)$ определяется зеемановским расщеплением уровней триона $\Delta E_{h h}=-3 g_{h} \mu_{\mathrm{B}} B \cos \theta$ с $g_{h}=-0.54$ (рис. 9,a).

В отличие от быстрого (за время меньшее $1 \mathrm{~ns}$ ) нарастания циркулярной поляризации $P_{c}(t)$ для ФЛ нейтральных экситонов, поляризация трионной ФЛ в малых полях устанавливается за время порядка $\tau_{s}^{\exp } \approx 60 \mathrm{~ns}$, значительно превышающее $\tau$. Медленное нарастание $P_{c}$ обусловлено процессами спиновой релаксации в отрицательно заряженном трионе, связанными с переворотом спинового момента $M= \pm 3 / 2$ тяжелых дырок. В результате в малых полях наблюдалась неравновесная степень поляризации $P_{c}^{\text {int }}$, значительно меньшая величины $P_{c}^{\mathrm{eq}}$. При увеличении магнитного поля спиновая релаксация ускорялась, и значение $P_{c}$ стремилось к $P_{c}^{\mathrm{eq}}$ (рис.9, $\left.a\right)$.

Ускорение спиновой релаксации в магнитном поле происходит за счет подмешивания дырок с проекциями $M= \pm 1 / 2$ к дыркам с проекциями $M= \pm 3 / 2$ поперечной к оси $c$ компонентой магнитного поля $B \sin \theta$. Скорость спиновой релаксации имеет вид [55]:

$$
\frac{1}{\tau_{s}(B, \theta)}=\frac{1}{\tau_{s 0}(T)}+\alpha(T) B^{2} \sin ^{2} \theta,
$$

где $\tau_{s 0}(T)$ - время спиновой релаксации в нулевом магнитном поле, а коэффициент $\alpha(T)$ описывает фононный механизм, который перебрасывает дырку из промежуточного состояния $M= \pm 1 / 2$ на второй подуровень основного состояния $M=\mp 3 / 2$. Температурная зависимость $1 / \tau_{s 0}, \alpha(T) \propto T^{2}$ позволила предположить, что переворот спина дырки связан преимущественно с двухфононными процессами [55].

Анализ экспериментальной зависмости $P_{c}^{\text {int }}(B)$ для ансамбля QD с помощью выражения (27) с $\rho_{\mathrm{ex}}=$ $=\rho_{h h}^{\mathrm{eq}}(B, x) /\left(1+\tau_{s}(B, x) / \tau\right)$ позволил определить время спиновой релаксации $\tau_{s}$ в индивидуальных QD в зависимости от угла $\theta$. Максимальная скорость спиновой релаксации достигается при $\theta \rightarrow 90^{\circ}$, однако такие QD
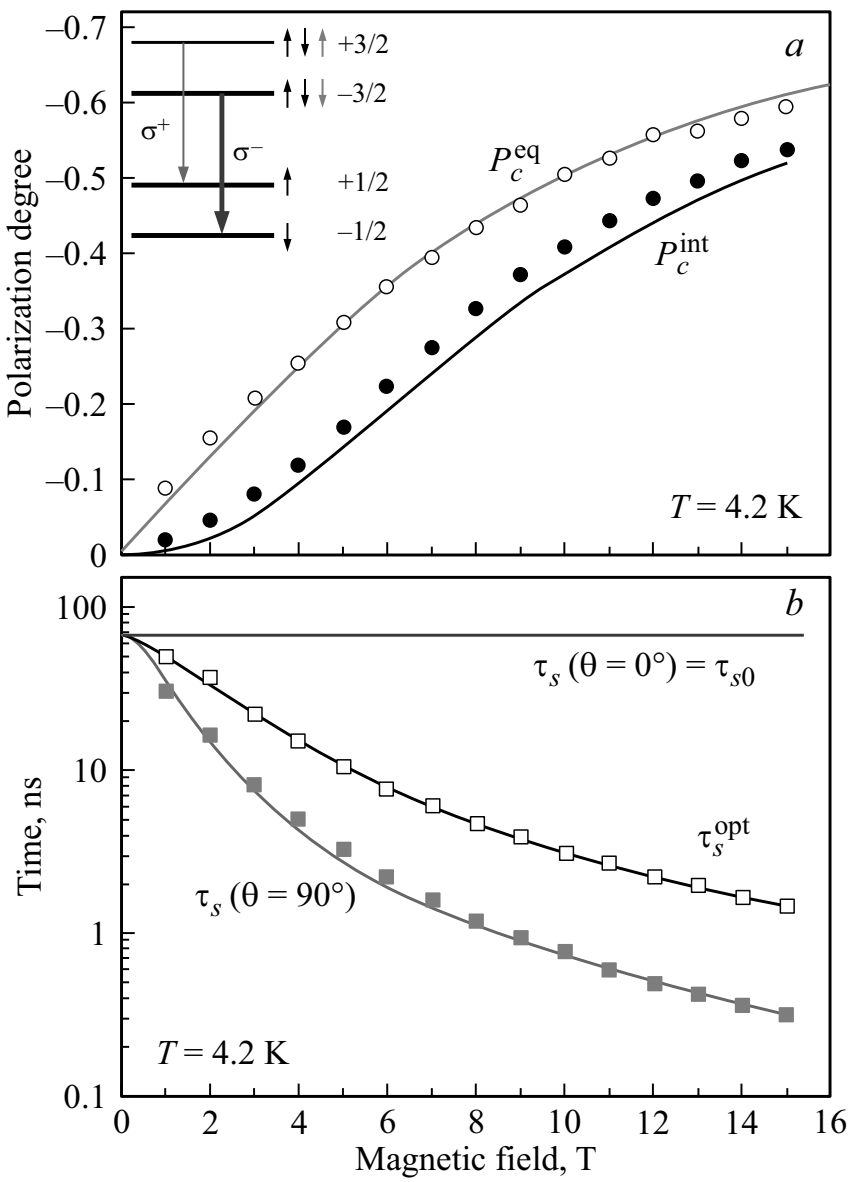

Рис. 9. (а) Зависимость равновесной $P_{c}^{\mathrm{eq}}$ и неравновесной $P_{c}^{\text {int }}$ степени циркулярной поляризации трионной ФЛ от магнитного поля в ансамбле произвольно ориентированных нанокристаллов $\mathrm{CdSe} / \mathrm{CdS}$ с радиусом ядра $2.5 \mathrm{~nm}$ и толщиной оболочки $10 \mathrm{~nm}$ [55]. На вставке показано схематичное изображение структуры спиновых уровней и оптических переходов в отрицательно заряженном трионе во внешнем магнитном поле. Тонкие и толстые короткие стрелки показывают направление спинов электрона и дырки соответственно. Циркулярно поляризованные оптические переходы $\sigma^{+}$и $\sigma^{-}$показаны длинными стрелками. (b) Зависимость времени спиновой релаксации от магнитного поля в нанокристаллах с осью $c$, ориентированной перпендикулярно $\left(\tau_{s}\left(\theta=90^{\circ}\right)\right)$ и параллельно $\left(\tau_{s}\left(\theta=0^{\circ}\right)\right)$ магнитному полю. $\tau_{s}^{\text {opt }}$ соответствует оптимально ориентированным НК, дающим основной вклад в неравновесную циркулярную поляризацию $P_{c}^{\text {int }}$.

не дают значительного вклада в $P_{c}^{\text {int }}$ из-за $\Delta E_{h h} \ll 1$. $\mathrm{B} \mathrm{QD}$, ориентированных параллельно магнитному полю, $\Delta E_{h h}$ максимально, однако спиновая релаксация остается медленной. На рис. 9, $b$ показаны зависимости $\tau_{s}(\theta)$ от магнитного поля для перпендикулярно $\left(\theta=90^{\circ}\right)$ и параллельно $\left(\theta=0^{\circ}\right)$ ориентированных QD. Время релаксации $\tau_{s}^{\text {opt }}$ описывает подгруппу оптимально ориентированных QD, которые дают основной вклад в $P_{c}^{\text {int }}$. Теоретическая зависимость $\tau_{s}^{\text {opt }}(B)$ с хорошей точностью воспроизводит экспериментально измеренную зависимость времени $\tau_{s}^{\exp }(B)$ нарастания $P_{c}(t)$ [55]. 
Выражение (27) может быть также обобщено для учета непроизвольной ориентации NC в ансамбле. Например, для $\mathrm{CdSe} / \mathrm{CdS} \mathrm{NPL}$ с толстой оболочкой наблюдалось бимодальное распределение ориентации $f_{\text {or }}(x)=n_{\text {hor }} \delta(1-x)+\left(1-n_{\text {hor }}\right) \delta(x)$, где $n_{\text {hor }}-$ доля горизонтально лежащих NPL. Уменьшение плотности NPL в ансамбле приводит к увеличению $n_{\text {hor }}$ и увеличению $\left|P^{\text {sat }}\right|=2 n_{\text {hor }} /\left(1+n_{\text {hor }}\right)[62]$.

\section{7. Заключение}

В настоящее время спиновая физика экситонов в полупроводниковых коллоидных нанокристаллах динамично развивается. Это связано с разработкой новых методов создания коллоидных нанокристаллов различной формы и химического состава, освоением новых методов характеризации нанокристаллов и открытием новых эффектов, таких как спиновый магнетизм немагнитных нанокристаллов. В настоящем обзоре показано, что спин-зависимые свойства экситонов в полупроводниковых коллоидных нанокристаллах являются ключом к пониманию процессов их рекомбинации. Представлена теория, описывающая механизм рекомбинации темного экситона путем одновременного переворота поверхностного спина и спина электрона в темном экситоне, приводящего к подмешиванию состояний светлого экситона. Магнетизм, вызванный поверхностными спинами, должен проявляться во всех типах коллоидных нанокристаллов, вне зависимости от их формы, химического состава и способа пассивации поверхности. Исследование данного эффекта может принести новые знания о взаимодействии экситонов с поверхностью. Важными направлениями для дальнейших исследований в спиновой физике коллодных нанокристаллов нам представляются проблемы спиновой динамики носителей и экситонных комплексов, включая их когерентую спиновую динамику.

Авторы благодарны всем коллегам, внесшим вклад в работы, представленные в этом обзоре: М. Bayer, A. Shabaev, M.A. Семиной, Н.А. Гиппиусу, В.Ф. Сапеге, Ю.Г. Кусраеву, L. Biadala, F. Liu, C. Javaux, B. Mahler, J.-P. Hermier, B. Siebers, A.A. Mitioglu, M.V. Ballottin, P.C.M. Christianen, T. Aubert, Z. Hens, M. Nasilowski и B. Dubertret. Особенно авторы признательны Ал.Л. Эфросу, внесшему большой вклад в развитие теории экситонной физики коллоидных нанокристаллов, многолетнее сотрудничество с которым стимулировало и поддерживало наш интерес к этим исследованиям.

\section{Список литературы}

[1] Е.Ф. Гросс, А.А. Каплянский. Оптика и спектроскопия 11, 204 (1957).

[2] А.И. Екимов, А.А. Онущенко, В.А. Цехомский. Физика и химия стекла 6, 511 (1980).
[3] А.И. Екимов, А.А. Онущенко. Письма в ЖЭТФ 34, 363 (1981).

[4] А.И. Екимов, А.А. Онущенко. Письма в ЖЭТФ 40, 337 (1984).

[5] R. Rossetti, S. Nakahara, L.E. Brus. J. Chem. Phys. 79, 1086 (1983).

[6] L.E. Brus. J. Chem. Phys. 80, 4403 (1984).

[7] M.V. Kovalenko, L. Manna, A. Cabot, Z. Hens, D.V. Talapin, C.R. Kagan, V.I. Klimov, A.L. Rogach, P. Reiss, D.J. Milliron, P. Guyot-Sionnnest, G. Konstantatos, W. Parak, T. Hyeon, B.A. Korgel, C.B. Christopher, W. Heiss. ACS Nano 9, 1012 (2015).

[8] A.C. Berends, C. de Mello Donega. J. Phys. Chem. Lett. 8, 4077 (2017).

[9] D.V. Talapin, J.S. Lee, M.V. Kovalenko, E.V. Shevchenko. Chem. Rev. 110, 389 (2010).

[10] O. Chen, J. Zhao, V.P. Chauhan, J. Cui, C. Wong, D.K. Harris, H. Wei, H.S. Han, D. Fukumura, R.K. Jain, M.G. Bawendi. Nature Mater. 12, 445 (2013).

[11] J. Bao, M.G. Bawemdi. Nature 523, 67 (2015).

[12] J. Owen, L. Brus. J. Am. Chem. Soc. 139, 10393 (2017).

[13] J. Owen. Science 347, 615 (2015).

[14] M.A. Boles, D. Ling, T. Hyeon, D.V. Talapin. Nat. Mater. 15, 141 (2016).

[15] M.S. Seehra, P. Dutta, S. Neeleshwar, Y.Y. Chen, C.L. Chen, S.W. Chou, C.C. Chen, C.L. Dong, C.L. Chang. Adv. Mater. 20, 1656 (2008).

[16] R.W. Meulenberg, J.R.I. Lee, S.K. McCall, K.M. Hanif, D. Haskel, J.C. Lang, L.J. Terminello, T. Van Buuren. J. Am. Chem. Soc. 131, 6888 (2009).

[17] L. Piveteau, T.C. Ong, A.J. Rossini, L. Emsley, C. Copéret, M.V. Kovalenko. J. Am. Chem. Soc. 137, 13964 (2015).

[18] A.J. Rossini, A. Zagdoun, M. Lelli, A. Lesage, C. Copéret, L. Emsley. Accounts Chem. Res. 46, 1942 (2013).

[19] Ал.Л. Эфрос, А.Л. Эфрос. ФТП 16, 1209 (1982).

[20] A.I. Ekimov, F. Hache, M.C. Schanne-Klein, D. Ricard, C. Flytzanis, I.A. Kudryavtsev, T.V. Yazeva, A.V. Rodina, Al.L. Efros. J. Opt. Soc. Am. B 10, 100 (1993).

[21] L. Keldysh. Phys. Status Solidi (a) 164, 3 (1993).

[22] A1.L. Efros, M. Rosen, M. Kuno, M. Nirmal, D. Norris, M. Bawendi. Phys. Rev. B 54, 4843 (1996).

[23] С.В. Гупалов, Е.Л. Ивченко. ФТТ 42, 1976 (2000).

[24] M.G. Bawendi, W.L. Wilson, L. Rothberg, P.J. Carroll, T.M. Jedju, M.L. Steigerwald, L.E. Brus. Phys. Rev. Lett. 65, 1623 (1990).

[25] M. Nirmal, C. Murray, M. Bawendi. Phys. Rev. B 50, 2293 (1994).

[26] M. Nirmal, D. Norris, M. Kuno, M. Bawendi, Al.L. Efros, M. Rosen. Phys. Rev. Lett. 75, 3728 (1995).

[27] M. Chamarro, C. Gourdon, P. Lavallard, O. Lublinskaya, A.I. Ekimov. Phys. Rev. B 53, 1336 (1996).

[28] A1.L. Efros. In: Semiconductor and metal nanocrystals: Synthesis and electronic and optical properties / Ed. by V.I. Klimov, M. Dekker. N.Y.(2003). 103-141 pp.

[29] M. Califano, A. Franceschetti, A. Zunger. Phys. Rev. B 75, 115401 (2007).

[30] M. Korkusinski, O. Voznyy, P. Hawrylak. Phys. Rev. B 82, 245304 (2010).

[31] A. Shabaev, Al.L. Efros. Nano Lett. 4, 1821 (2004). 
[32] L. Biadala, F. Liu, M.D. Tessier, D.R. Yakovlev, B. Dubertret, M. Bayer. Nano Lett. 14, 1134 (2014).

[33] L. Biadala, B. Siebers, Y. Beyazit, M.D. Tessier, D. Dupont, Z. Hens, D.R.Yakovlev, M. Bayer. ACS Nano 10, 3356 (2016).

[34] A. Rodina, Al.L. Efros. Nano Lett. 15, 4214 (2015).

[35] A. Rodina, A1.L. Efros. Phys. Rev. B 93, 155427 (2016).

[36] В.И. Перель, Б.П. Захарченя. В сб.: Опитическая ориентация / Под ред. Б.П. Захарченя, Ф. Майер. Наука, Л. (1989), Гл. 1. С. $11-16$.

[37] Е.Л. Ивченко. ФТТ 60, 8, 1471 (2018).

[38] С.Ю. Вербин, С.А. Пермогоров, А.Н. Резницкий. ФТТ 25, 346 (1983).

[39] S. Schmitt-Rink, D.A.B. Miller, D.S. Chemla. Phys. Rev. B 35, 8113 (1987).

[40] E. Yablonovitch, T.J. Gmitter, R. Bhat. Phys. Rev. Lett. 61, 2546 (1988).

[41] Л.Д. Ландау, Е.М. Лифшиц. Электродинамика сплошных сред. Наука, М., (1992).

[42] M.G. Bawendi, P.J. Carroll, W.L. Wilson, L.E. Brus. J. Chem. Phys. 96, 946 (1992).

[43] B. Siebers, L. Biadala, D.R. Yakovlev, A.V. Rodina, T. Aubert, Z. Hens, M. Bayer. Phys. Rev. B 91, 155304 (2015).

[44] A. Rodina, Al.L. Efros, ЖЭТФ 122, 641 (2016).

[45] E. Johnston-Halperin, D. Awschalom, S. Crooker, A.L. Efros, M. Rosen, X. Peng, A. Alivisatos. Phys. Rev. B 63, 205309 (2001)

[46] S.A. Crooker, T. Barrick, J.A. Hollingsworth, V.I. Klimov. Appl. Phys. Lett. 82, 2793 (2003).

[47] O. Labeau, P. Tamarat, B. Lounis. Phys. Rev. Lett. 90, 257404 (2003).

[48] M. Furis, J.A. Hollingsworth, V.I. Klimov, S.A. Crooker. J. Phys. Chem. B 109, 15332 (2005).

[49] C. De Mello Donegá, M. Bode, A. Meijerink. Phys. Rev. B 74, 085320 (2006).

[50] D. Oron, A. Aharoni, C. De Mello Donega, J. Van Rijssel, A. Meijerink, U. Banin. Phys. Rev. Lett. 102, 177402 (2009).

[51] L. Biadala, Y. Louyer, P. Tamarat, B. Lounis. Phys. Rev. Lett. 103, 037404 (2009).

[52] L. Biadala, Y. Louyer, P. Tamarat, B. Lounis. Phys. Rev. Lett. 105, 157402 (2010).

[53] I. Moreels, G. Rainò, R. Gomes, Z. Hens, T. Stöferle, R.F. Mahrt. ACS Nano 5, 8033 (2011).

[54] J.H. Blokland, V.I. Claessen, F.J.P. Wijnen, E. Groeneveld, C. de Mello Donegá, D. Vanmaekelbergh, A. Meijerink, J.C. Maan, P.C.M. Christianen. Phys. Rev. B 83, 035304 (2011).

[55] F. Liu, L. Biadala, A.V. Rodina, D.R. Yakovlev, D. Dunker, C. Javaux, J.P. Hermier, A.L. Efros, B. Dubertret, M. Bayer. Phys. Rev. B 88, 035302 (2013).

[56] F. Liu, A.V. Rodina, D.R. Yakovlev, A. Greilich, A.A. Golovatenko, A.S. Susha, A.L. Rogach, Y.G. Kusrayev, M. Bayer. Phys. Rev. B 89, 115306 (2014).

[57] L. Biadala, B. Siebers, R. Gomes, Z. Hens, D.R. Yakovlev, M. Bayer. J. Phys. Chem. C 118, 22309 (2014).

[58] L. Biadala, E. Shornikova, A. Rodina, D. Yakovlev, B. Siebers, N. Aubert, M. Nasilowski, Z. Hens, B. Dubertret, A. Efros, M. Bayer. Nature Nanotechnology 12, 569-574 (2017).
[59] E.V. Shornikova, L. Biadala, D.R. Yakovlev, V.F. Sapega, K.Y. G., A.A. Mitioglu, M.V. Ballottin, P.C.M. Christianen, V.V. Belykh, M.V. Kochiev, N.N. Sibeldin, A.A. Golovatenko, A.V. Rodina, N.A. Gippius, A. Kuntzmann, J. Ye, M. Nasilowski, B. Dubertret, M. Bayer. Nanoscale 10, 646 (2018).

[60] G. Pettinari, E. Groeneveld, C. De Mello Donegá, D. Vanmaekelbergh, J.C. Maan, P.C.M. Christianen. J. Phys. Chem. C 121, 23693 (2017).

[61] C. Javaux, B. Mahler, B. Dubertret, A. Shabaev, A.V. Rodina, A.L. Efros, D.R. Yakovlev, F. Liu, M. Bayer, G. Camps, L. Biadala, S. Buil, X. Quelin, J.P. Hermier. Nature Nanotechnology 8, 206 (2013).

[62] E.V. Shornikova, L. Biadala, D.R. Yakovlev, D.H. Feng, V.F. Sapega, N. Flipo, A.A. Golovatenko, M.A. Semina, A.V. Rodina, A.A. Mitioglu, M.V. Ballotin, P.C.M. Christianen, Y.G. Kusraev, M. Nasilowski, B. Dubertret, M. Bayer. Nano Lett. 18, 373-380 (2018).

[63] A. Shabaev, A.V. Rodina, Al.L. Efros. Phys. Rev. B 86, 205311 (2012).

[64] U. Woggon, F. Gindele, O. Wind, C. Klingshirn. Phys. Rev. B 54, 1506 (1996).

[65] D. Norris, Al.L. Efros, M. Rosen, M. Bawendi. Phys. Rev. B 53, 16347 (1996).

[66] F. Wijnen, J. Blokland, P. Chin, P. Christianen, J. Maan. Phys. Rev. B 78, 235318 (2008).

[67] A. Granados Del Águila, B. Jha, F. Pietra, E. Groeneveld, C. De Mello Donegá, J.C. Maan, D. Vanmaekelbergh, P.C.M. Christianen. ACS Nano 8, 5921 (2014).

[68] N. Le Thomas, E. Herz, O. Schöps, U. Woggon, M. Artemyev. Phys. Rev. Lett. 94, 016803 (2005).

[69] Y. Louyer, L. Biadala, J.B. Trebbia, M.J. Fernée, P. Tamarat, B. Lounis. Nano Lett. 11, 4370 (2011).

[70] Y. Louyer, L. Biadala, P. Tamarat, B. Lounis. Appl. Phys. Lett. 96, 203111 (2010).

[71] M.J. Fernée, P. Tamarat, B. Lounis J. Phys. Chem. Lett. 4, 609 (2013).

[72] M. Califano, A. Franceschetti, A. Zunger. Nano Lett. 5, 2360 (2005).

[73] K. Leung, S. Pokrant, K.B. Whaley. Phys. Rev. B 57, 12291 (1998).

[74] S. Goupalov. Phys. Rev. B 74, 113305 (2006).

[75] P.C. Sercel, A. Shabaev, Al.L. Efros. Nano Lett. 17, 4820 (2017).

[76] G. Mackh, W. Ossau, D.R. Yakovlev, A. Waag, G. Landwehr, R. Hellmann, E.O. Göbel. Phys. Rev. B 49, 10248 (1994).

[77] D.R. Yakovlev, W. Ossau. In: Introduction to the physics of diluted magnetic semiconductors / Ed. J. Kossut, J.A. Gaj. Springer, Berlin (2010). Ch. 7. 221 p.

[78] S.J. Cheng, P. Hawrylak. Eur. Phys. Lett. 81, 37005 (2008).

[79] F. Qu, P. Hawrylak. Phys. Rev. Lett. 96, 157201 (2006).

[80] R.M. Abolfath, P. Hawrylak, I. Žutić. Phys. Rev. Lett. 98, 207203 (2007).

[81] D.A. Bussian, S.A. Crooker, M. Yin, M. Brynda, Al.L. Efros, V.I. Klimov. Nat. Mater. 8, 35 (2009).

[82] R. Viswanatha, J.M. Pietryga, V.I. Klimov, S.A. Crooker. Phys. Rev. Lett. 107, 067402 (2011).

[83] I.A. Merkulov, A.V. Rodina. In: Introduction to the physics of diluted magnetic semiconductors / Ed. by J. Kossut, J.A. Gaj. Springer, Berlin (2010). Ch. 3. Pp. 65-101.

[84] R. Beaulac, L. Schneider, P.I. Archer, G. Bacher, D.R. Gamelin. Science 325, 973 (2009). 
[85] H.D. Nelson, L.R. Bradshaw, C.J. Barrows, V.A. Vlaskin, D.R. Gamelin. ACS Nano 9, 11177 (2015).

[86] W.D. Rice, W. Liu, T.A. Baker, N.A. Sinitsyn, V.I. Klimov, S.A. Crooker. Nat. Nanotechnol. 11, 137 (2016).

[87] W.D. Rice, W. Liu, V. Pinchetti, D.R. Yakovlev, V.I. Klimov, S.A. Crooker. Nano Lett. 17, 3068 (2017).

[88] F. Muckel, C.J. Barrows, A. Graf, A. Schmitz, C.S. Erickson, D.R. Gamelin, G. Bacher. Nano Letters 17, 4768 (2017).

[89] Г.Л. Бир, Г.Е. Пикус. Симметрия и деформационные эффекты в полупроводниках. Наука, М. (1972).

[90] E.I. Ivchenko, G.E. Pikus. Superlattices and other heterostucture. Springer, Berlin. (1997).

[91] A1.L. Efros. Phys. Rev. B 46, 7448 (1993).

[92] Al.L. Efros, A.V. Rodina. Phys. Rev. B 47, 10005 (1993).

[93] M.A. Semina, A.A. Golovatenko, A.V. Rodina. Phys. Rev. B 93, 045409 (2016).

[94] V.P. Kochereshko, G.V. Mikhailov, I.N. Uraltsev. Sov. Phys. Solid State 25, 439 (1983), [ФTT 25, 769-776 (1983)].

[95] E.I. Ivchenko. Optical spectroscopy of semiconductor nanostructures, Alpha Science International Ltd., Harrow, U.K. (2005).

[96] А.И. Ансельм. Введение в теорию полупроводников. Наука, М., (1978).

[97] C. Lethiec, J. Laverdant, H. Vallon, C. Javaux, B. Dubertret, J.M. Frigerio, C. Schwob, L. Coolen, A. Maitre. Phys. Rev. X 4, 021037 (2014).

[98] S.A. Empedocles, R. Neuhauser, K. Shimizu, M.G. Bawendi. Adv. Mater. 11, 1243 (1999).

[99] I. Chung, K.T. Shimizu, M.G. Bawendi. Proc. Natl. Acad. Sci. U.S.A. 100, 405 (2003).

[100] В.В. Батыгин, И.Н. Топтыгин. Сборник задач по электродинамике. Наука, М. (1970).

[101] Al.L. Efros, A.I. Ekimov, F. Kozlowski, V. Petrova-Koch, H. Schmidbaur, S. Shumilov. Solid State Commun. 78, 853 (1991).

[102] С.В. Гупалов, И.А. Меркулов. ФТТ 41, 1473 (1999).

[103] A. Rodina, A. Golovatenko, E. Shornikova, D. Yakovlev, A1.L. Efros. Semiconductors 52, 572 (2018).

[104] P.G. de Gennes. Phys. Rev. 118, 141 (1960).

[105] Э.Л. Нагаев, Письма в ЖЭТФ 6, 484 (1967).

[106] I.A. Merkulov, D.R. Yakovlev, A. Keller, W. Ossau, J. Geurts, A. Waag, G. Landwehr, G. Karczewski, T. Wojtowicz, J. Kossut. Phys. Rev. Lett. 83, 1431 (1999).

[107] М.И. Дьяконов, В.И. Перель, Письма в ЖЭТФ 16, 563 (1972).

[108] В.Л. Коренев. Письма в ЖЭТФ 70, 129 (1999).

[109] M. Nirmal. Photophysics of CdSe semiconductor nanocrystals MIT, (1996).

[110] J.R. Murphy, S. Delikanli, T. Zhang, T.A. Scrace, P. Zhang, T. Norden, T. Thomay, A.N. Cartwright, H.V. Demir, A. Petrou. Proc. SPIE 10114, 101140R-101140R-11 (2017).

[111] I. Broser, R. Broser, A. Hoffmann. In Landoldt-Börnstein Tables. New Series. Group III: Crystals and Solid State Physics / Ed. O. Madelung Springer, Berlin. (1982).

[112] J.A. Gupta, D.D. Awschalom, Al.L. Efros, A.V. Rodina. Phys. Rev. B 66, 125307 (2002).

[113] A.V. Rodina, Al.L. Efros, A.Y. Alekseev. Phys. Rev. B 67, 155312 (2003).

Редактор Ю.Э. Китаев 